\title{
Excitation of Periodical Shock Waves in Solid-State Optical Media (Yb:YAG, Glass) at SBS of Focused Low-Coherent Pump Radiation: Structure Changes, Features of Lasing
}

\author{
N.E. Bykovsky and Yu.V. Senatsky \\ Lebedev Physical Institute, Russian Academy of Sciences, Moscow \\ Russia
}

\section{Introduction}

During several last decades much attention was paid to the processes that occur in solidstate optical media under the interaction with high-power focused laser radiation. A great number of studies were devoted to the phenomena of optical breakdown, structure changes, stimulated scatterings, generation of hypersonic waves in transparent dielectrics under the action of nanosecond (ns) and picosecond (ps) laser pulses (Manenkov \& Prokhorov, 1986; Nelson et al., 1982; Ready, 1971; Robinson et al., 1984; Stuart et al., 1995). Recent interest in these studies was stimulated by the appearance of lasers with femtosecond (fs) pulses (Gordienko et al., 2010; Merlin, 1997; Sakakura et al., 2007).

An experimental study of a small region with high pressure and temperature gradients formed in a medium at focusing high-power laser radiation had been performed, as a rule, outside the laser cavity. In our experiment (Basiev et al., 2004), a region with such properties happened to be formed directly in the 2-mirror laser cavity, when Yb:YAG samples were pumped by the focused wide-band $(0,89-0,95 \mu \mathrm{m})$ radiation from a pulsed LiF: $\mathrm{F}_{2}{ }^{+}$color center laser (ccl). Thus, in contrast to many studies on ytterbium lasers, conditions for generation in $\mathrm{Yb}$-doped samples in this experiment had been distinguished by the very high intensity (over $1 \mathrm{GW} / \mathrm{cm}^{2}$ ) of the pump, which moreover had a low coherence. Experiments on pumping of $\mathrm{Yb}$-doped and non-doped samples of different optical media (YAG, glass, $\mathrm{LiF}$ et al.) by powerful low-coherent radiation from $\mathrm{LiF}$ : $\mathrm{F}_{2}{ }^{+} \mathrm{ccl}$ were continued in subsequent papers (Bykovsky, 2005, 2006; Bykovsky \& Senatsky, 2008a,b, 2010). At intensities $I \geq 1 \mathrm{GW} / \mathrm{cm}^{2}$ interaction of ccl pump radiation with the medium in the focal region was essentially nonlinear. The interaction of ccl pulses with samples was accompanied by excitation of stimulated Brillouin scattering (SBS) and stimulated Raman scattering (SRS) of pump radiation. The scattering generated hypersonic waves of high amplitude, which were converted into a periodic sequence of shock waves with sharp pressure jumps on their fronts propagating along the direction of pump. Pressure jumps were so large that they caused a phase transition in an optical medium, which was observed near the sample surface in the form of small domains with spatial modulation of the refractive index caused by the interference of hypersonic waves. 
Under ccl pumping due to heat release and generation of intensive hypersonic waves a region with strong temperature, pressure and refractive index gradients and at the same time with a high-level of inversion was formed in the focus of the pump laser in Yb-doped materials. Despite the strong optical inhomogeneity of the medium, Yb lasing in 10-15 ns pulses was observed in $\mathrm{Yb}$ :YAG (with $20 \%$ concentration of $\mathrm{Yb}^{3+}$ ions) and $\mathrm{Yb}$ :glass (with $10 \% \mathrm{Yb}^{3+}$ concentration) during the action of the 20-30 ns pump pulse and after it (due to inversion remaining in the medium). During the SBS of pump radiation the hypersonic wave spatial structure served as a resonator for $\mathrm{Yb}$ lasing. Shock waves (with phonon energies up to $1000 \mathrm{~cm}^{-1}$ ) affected the generation dynamics. The $\mathrm{Yb}$ lasing was distinguished by some specific characteristics such as a surprisingly wide spectrum (up to $50 \mathrm{~nm}$ ) and a high directivity of the emission. In addition to the wide-band generation on the shock-waves grating there was also observed $\mathrm{Yb}$ lasing on resonator modes. After the end of the pump pulse another sequence of shock waves diverging outward the focal region affected the build-up of generation between 2 mirrors in the cavity. The line spectra of $\mathrm{Yb}$ generation in the resonator contained twisted spectral lines with structures of small-scale spots.

Description of these unusual phenomena observed under the interaction of short intense pump laser pulse of low coherence with optical media and their explanation are presented in this chapter. The optical scheme and the parameters of $\mathrm{LiF}: \mathrm{F}_{2}{ }^{+} \mathrm{ccl}$ are considered. The features of SBS and SRS and the appearance of the periodical shock waves in the optical medium at low-coherent pumping are discussed. The interpretation to observed specific optical damage is given. The mechanism of generation of broadband, high-directional short laser pulses in the spatial structure of thin layers with inversion produced in the region of propagation of intense hypersonic waves in the medium is discussed. Conditions for generation in a 2 mirror resonator containing active medium with a strong refractive index gradient are considered. The interpretation of the observed twisted lines with small-scale structures in generation spectra as well as temporal, spatial-angular characteristics of $\mathrm{Yb}$ lasing in the resonator is given.

\section{LiF: $\mathrm{F}_{2}{ }^{+}$color center pump laser}

Optical pumping of $\mathrm{Yb}$-doped materials can be performed only into the single $\mathrm{Yb}^{3+}$ ion absorption band at ${ }^{2} \mathrm{~F}_{7 / 2}-{ }^{2} \mathrm{~F}_{5 / 2}$ transition near $0.9 \mu \mathrm{m}$ (Figs.1, 2). In this connection, the use of flash lamps as sources of broadband radiation for pumping an Yb-doped medium is ineffective. At the present moment, the most effective and widely used sources of $\mathrm{Yb}$-doped materials pumping are semiconductor laser diodes operating within the spectral range near $0.94 \mu \mathrm{m}$. Along with semiconductor diodes solid-state laser pump sources have been used to investigate Yb-doped active media. Cr:LiSAF, Ti:Sa, Nd:YAG (0.94 $\mu \mathrm{m}$ transition) lasers have been used to pump Yb:S-FAP and Yb:YAG (Bykovsky et al., 2000; Kanabe et al., 2000; Marshall et al., 1997, as cited in Bykovsky \& Senatsky, 2008b).

In our work a LiF: $\mathrm{F}_{2}{ }^{+}$color center laser (ccl) was used to pump an Yb:YAG crystal (Basiev et al., 2004). The lithium fluorine color center (LiF: $\mathrm{F}_{2}{ }^{+}, \mathrm{LiF}: \mathrm{F}_{2}{ }^{-}$) lasers are the sources of radiation in the near IR $(0.8-1.3 \mu \mathrm{m})$, and they effectively convert the neodymium and ruby laser radiation into this spectral range (Basiev et al., 1982). Possible room temperature operation, high conversion efficiency (up to $30 \%$ ), and a large generation tuning range (more than $1000 \mathrm{~cm}^{-1}$ ) make such lasers very attractive for certain practical applications. The 
pumping of $\mathrm{Yb}$-doped samples by the ccl had been, undoubtedly, inferior to laser diodes pumping in efficiency. However, at focusing $\mathrm{ccl}$ radiation in the Yb-doped medium the pump power densities typical of semiconductor diodes could be easily exceeded. This had been, of course, of interest for research. Therefore, just the ccl had been used later in experiments on study lasing in $\mathrm{Yb}$-doped samples as well as nonlinear interaction of pump radiation with optical materials. The possible generation region of $\mathrm{LiF}: \mathrm{F}_{2}{ }^{+} \mathrm{ccl}$ extends from 0.83 to $1.1 \mu \mathrm{m}$ (Basiev et al., 1982) and completely covers the absorption band of Yb:YAG. In particular experimental conditions the actual emission band of ccl depends on the selective properties of the resonator, and usually covers only part of the noted wavelength range.

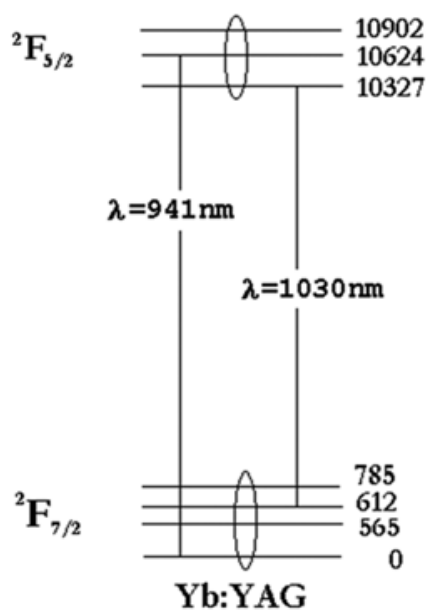

Fig. 1. Energy level diagram of $\mathrm{Yb}^{3+}$ ions in a $\mathrm{Yb}$ :YAG crystal. Ovals combine the Stark components of levels with rapid $\left(\approx 10^{-12} \mathrm{~s}\right)$ thermal relaxation (Krupke, 2000)

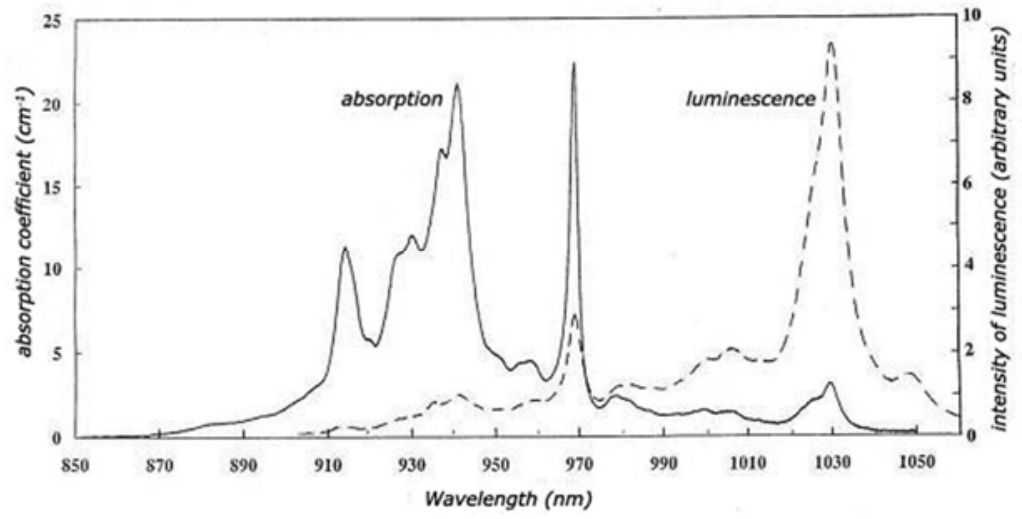

Fig. 2. Absorption and luminescence spectra of $\mathrm{Yb}^{+3}$ ions in the YAG crystal $(1 \mathrm{~mm}$ plate, $\mathrm{Yb}^{+3}$ ions concentration $20 \%$ ) 
Figure 3 illustrates the scheme of the $\mathrm{ccl}$ with elements of radiation transport and diagnostics. A LiF: $\mathrm{F}_{2}{ }^{+}$crystal $(40 \times 20 \times 6 \mathrm{~mm})$ was placed in the resonator formed by a plane mirror $\mathrm{M}_{1}(\approx 100 \%$ reflection at $0.9 \mu \mathrm{m})$ and a glass plate $\mathrm{M}_{2}$. The length of the ccl resonator made $30 \mathrm{~cm}$. The LiF: $\mathrm{F}_{2}{ }^{+}$crystal was pumped through glass plate by a ruby laser operating in the single shot regime with pulse duration $\approx 30 \mathrm{~ns}$ and the energy up to $1 \mathrm{~J}$. A multimode radiation at the wavelength of $0.694 \mu \mathrm{m}$ was focused into the $\mathrm{LiF}: \mathrm{F}_{2}{ }^{+}$crystal by a lens $\mathrm{L}_{1}$ with the focal length $F_{1}=500 \mathrm{~mm}$. The ccl multimode radiation was, in its turn, focused on the studied samples by a lens $\mathrm{L}_{2}$ with the focal length $\mathrm{F}_{2}=120 \mathrm{~mm}$. The $\mathrm{Yb}$-doped samples were placed into a compact two-mirror resonator.

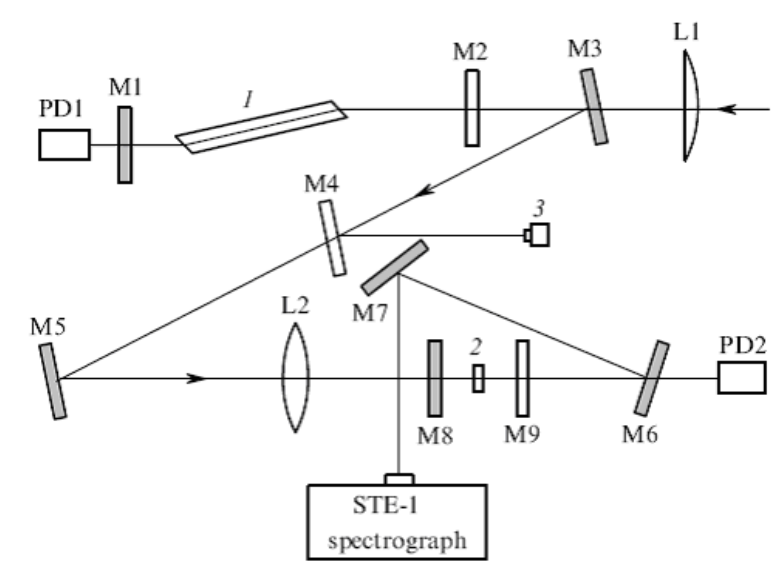

Fig. 3. Scheme of the experimental setup: (1) active element of a ccl; (2) Yb-dopped plate in the resonator with mirrors M8 and M9; (3) calorimeter; (M1, M2) ccl resonator mirrors; (M3 M7) steering mirrors for ccl and Yb laser radiation; (L1 - L2) lenses; (PD1, PD2) photodiodes

The ruby laser and ccl pulses were registered with the help of photodiodes and a twochannel oscilloscope, and the laser energy was measured by calorimeters. The ccl radiation spectra were analyzed by the STE-1 spectrograph operating in the near IR region. The ccl emitted pulses of 20-30 ns duration and the energy of 100-150 mJ. For the most cases, the ccl pulse shape repeated the shape of the ruby laser pulse, Fig. 4a. Since the ccl resonator round-trip time made $\approx 2 \mathrm{~ns}$, then during the ruby laser pumping the radiation made not more than 15 round trips in the ccl cavity. Due to high amplification in the LiF: $\mathrm{F}_{2}{ }^{+}$medium the ccl pulse was formed in several round trips inside the resonator. Though the LiF: $\mathrm{F}_{2}{ }^{+}$ crystal was cut at a Brewster angle the ccl radiation was weakly polarized.

Figure $4 \mathrm{~b}$ presents the densitograms of $\mathrm{LiF}: \mathrm{F}_{2}{ }^{+}$laser spectrum. The $\mathrm{LiF}: \mathrm{F}_{2}{ }^{+}$laser emission was observed within the range $0.89-0.95 \mu \mathrm{m}$. The lines of an argon spectral lamp were used as the wavelengths markers. Large spectrum width of $\mathrm{ccl}$ and short time of radiation development in the resonator speak about low coherency of the ccl emission. The ccl multimode radiation divergence was $\approx 2 \times 10^{-3} \mathrm{rad}$. This allowed focusing the ccl pump at the sample into $\mathrm{a} \approx 250 \mu \mathrm{m}$ spot. Moving samples along the axis of the focused pump beam one could change the size of the focal region within the limits of $250 \div 1000 \mu \mathrm{m}$, and the power density in the medium within the range of $0.5 \div 5 \mathrm{GW} / \mathrm{cm}^{2}$. Such a range of power density 
variation allowed one to carry out both experiments on $\mathrm{Yb}$ lasing (see Section 4-6) and experiments on nonlinear interaction of ccl radiation with optical materials (see Section 3). In some of experiments, the ccl energy density was close to the damage threshold of Yb:YAG, glass and other studied materials. The material being damaged, the further experiments used a fresh part of a sample.

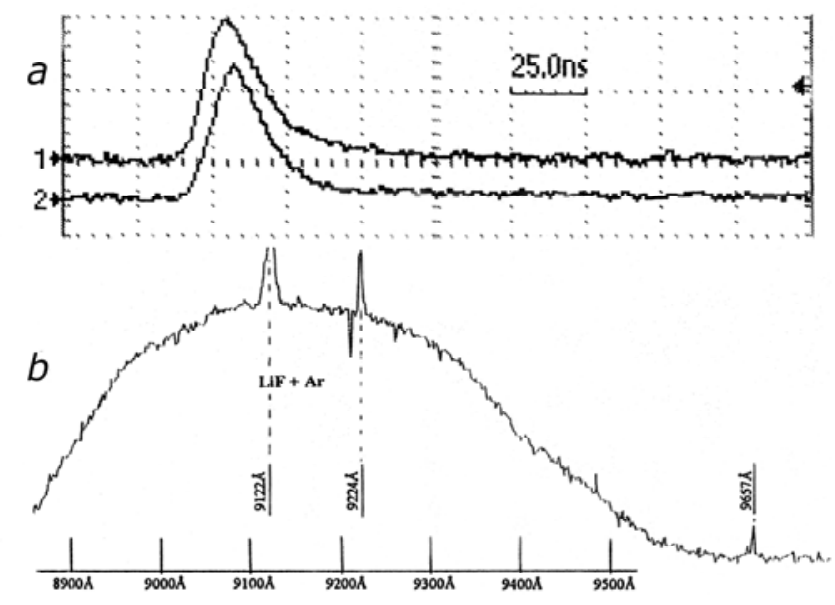

Fig. 4. Oscillograms of the ruby laser (1) and ccl (2) pulses - (a); densitograms of the LiF: $\mathrm{F}_{2}{ }^{+}$ laser spectrum with the argon spectral lamp reference lines - (b)

\section{Interaction of $\mathrm{ccl}$ radiation with optical media}

The experiments on nonlinear interaction of ccl radiation with optical media have been mainly performed using non-doped samples out of the cavity, Fig. 5. The ccl radiation was focused at samples by $L_{2}, F=120 \mathrm{~mm}$, and, so, within the sample thickness of 1-3 $\mathrm{mm}$ the pump power density changed insignificantly. The lens was tilted at $10^{0}$ to the direction of ccl beam so that reflections from lens surfaces would not come back to the ccl resonator. As the samples there were used plates and slabs with polished surfaces made of the following materials: crystalline quartz $(10 \times 10 \times 20 \mathrm{~mm})$, Yb:YAG crystal (1-2 mm thick plates), YAG crystal $(4,5 \times 30 \times 30 \mathrm{~mm})$, calcite $(2 \mathrm{~mm}$ plates), LiF crystal $(5 \mathrm{~mm}$ plate), $2 \mathrm{~mm}$ plexiglas plates, glass cube $(20 \times 20 \times 30 \mathrm{~mm})$. The ccl radiation was directed onto samples at a normal or at some angle (including the Brewster angle) to the sample's surface. The ccl pulse energy coming to samples varied within the range from 50 to $120 \mathrm{~mJ}$.

In all materials a strong scattering of radiation was registered under the action of a ccl pulse. A diagnostic complex consisting of photodiodes, oscilloscopes, calorimeters and a spectrograph was arranged on the stand (Fig.5) in order to study the scattering of the low coherent wideband $(0,89-0,95 \mu \mathrm{m})$ radiation of $\mathrm{LiF}: \mathrm{F}_{2}{ }^{+} \mathrm{ccl}$. Due to very large spectrum width (tens of $\mathrm{nm}$ ) and complicated space-angular structure of the scattered radiation components, one comes across difficulties in obtaining the spectral data on the scattered radiation, and it was not done. Strong scattering from the ccl focusing region in a wide angular range (tens of degrees) in forward and opposite directions (relative to the ccl beam) was observed for all 
samples at the ccl power density $\geq 1 \mathrm{GW} / \mathrm{cm}^{2}$. The scattering intensity grew with the growth of the ccl intensity. The strongest scattering was observed at an angle of $180^{\circ}$, i.e. back to the ccl aperture. The scattering was also observed at $90^{\circ}$ to the ccl beam. Using photodiodes FD with $\approx 1$ ns time resolution, the shape and duration of the ccl pulse and scattered radiation were registered by two-channel oscilloscopes of 1 ns and 4 ns resolution. To collect the scattered radiation to photodiodes the spherical mirrors were used, Fig. 5. The registered time and space-angular characteristics of scattering evidenced the rise of stimulated scattering (SBS and SRS) of wideband low-coherent ccl radiation in the samples.

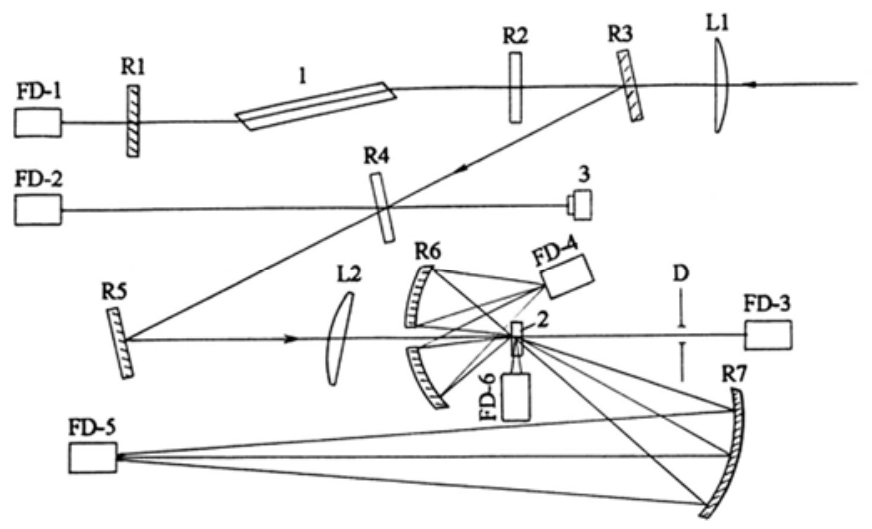

Fig. 5. Scheme of the experiment on the study of nonlinear phenomena in the crystal and glass samples: R1, R2 - LiF: $\mathrm{F}_{2}{ }^{+} \mathrm{ccl}$ mirrors; $\mathrm{R} 3$ - $\mathrm{R} 5$ ccl radiation transport mirrors; R6 - R7 mirrors to collect scattered radiation at diagnostic system; L1, L2 - lenses; FD - photodiodes; 1 - LiF: $\mathrm{F}_{2}{ }^{+}$active element; 2 - sample; 3 - calorimeter; $\mathrm{D}$ - diaphragm

Figure 6 shows oscillograms of the $\mathrm{ccl}$ and scattered radiation pulses. A series of oscillograms in Fig.6 (left) illustrates the SBS of ccl pump radiation in YAG. Strong back scattering typical of SBS was observed in the YAG sample, placed at different positions to the pump. The duration of backscattered pulses was always smaller than the pump pulse duration $(20 \div 30 \mathrm{~ns})$ and constituted $8 \div 15 \mathrm{~ns}$. At the oscillogram of the ccl pulse which passed the sample and the diaphragm D, one observes a pump «cut-off» typical for SBS, Fig. 6 (left, I, b). This pulse «cut-off» was connected with the reflection of the ccl beam incident onto the sample under the occurrence of a periodic grating formed by hypersonic waves.

Figure 6 (right) illustrates oscillograms of scattering in the YAG and crystalline quartz samples in forward and backward directions, as well as at $90^{\circ}$ to the ccl beam. Mirror R6 (Fig.5) focused the backward scattering at angles from $6^{0}$ to $43^{\circ}$, and mirror R7 - forward scattering at $5^{0}$ to $25^{0}$ in respect to the pump beam. The photodiode FD 6 recorded the $90^{\circ}$ scattering. The data obtained on the scattering of wide-band low-coherent ccl radiation allow one to consider the pulses observed as different forms of the stimulated scattering. The scattering backward to the ccl aperture proves to be the SBS of pump, as noted above. The forward scattering at large angles may be attributed to the anti-Stokes component of SRS, and the backward scattering at large angle is due to the Stokes component of SRS. Scattering at $90^{\circ}$, apparently, is due to the SBS and SRS of pump in the transverse direction. 
Profiles of the scattered pulses observed in the oscilloscope, Fig.6 (right) illustrate the dynamics of SBS and SRS in samples and the influence of different components of stimulated scattering on each other. It should be noted that the pump intensities at which SBS and SRS were observed in our experiments $\left(0.5 \mathrm{GW} / \mathrm{cm}^{2}\right)$, had been almost an order lower than those typical of scattering at a coherent pumping.
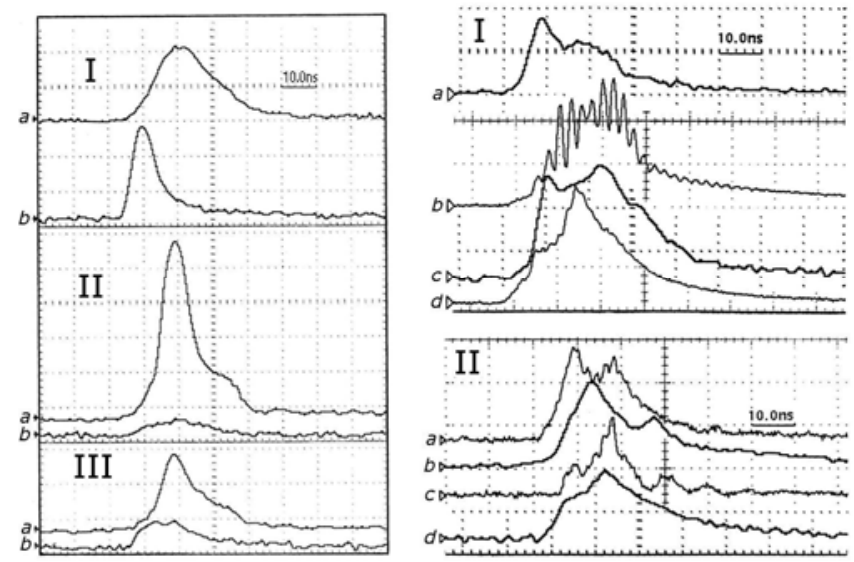

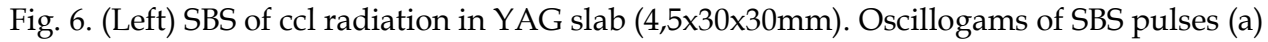
and ccl pulses behind the diaphragm $\mathrm{D}(\mathrm{b})$ for different orientations of the slab: I - pumping along the slab long side; II - pump beam along the short side; III - sample at Brewster angle to the pump. (Right) Oscillograms of scattering for YAG (I) and crystalline quartz (II): aSBS pulse; b-scattering at $90^{\circ}$ to the pump; c-anti-Stokes SRS signal, d-Stokes SRS signal

After the action of ccl pulses of $4-5 \mathrm{GW} / \mathrm{cm}^{2}$ intensity onto samples multiple marks of such an action in the form of volume and surface damages, and changes of the material structure were observed. Microphotographs of structural changes were collected, data were systematized. The material of transparent dielectrics was found to be damaged in different ways. Cracks in the medium, local regions of structure changes, tracks of self-focusing, and some other types of optical damage were observed near the surface and in the depth of a sample in the direction of the pump beam. Figures 7-9 demonstrate microphotographs of structural changes in YAG and LiF samples. One can observe plane formations of several micron thicknesses with a sharp boundary, Figs.7, 9. Most of such objects were concentrated near the sample surface at depths $<200 \mu \mathrm{m}$. Fig.8 demonstrates cracking at the LiF crystal surface. All these figures are illustrative for the energy release in the subsurface layers of samples. The optical damage in transparent dielectrics caused by high-power radiation was observed and studied for a few decades. The structure changes observed in the ccl experiments were compared with literature on the interaction of highly coherent radiation with transparent media. It was found that the low-coherent $\mathrm{ccl}$ radiation causes structural changes of some new kinds, which are not described in literature, for example, flat regions with structural changes bordered by a sharp boundary. The effects of nonlinear interaction of ccl pump radiation with transparent optical media (stimulated scattering, structural changes) were interpreted under the assumption that the SBS of low-coherent ccl radiation causes a succession of hypersonic shock waves. The formation of hypersonic shock waves at 
elastic nonlinearity at SBS was considered theoretically (Polyakova, 1966, 1968). But those results have not been so far supported by SBS experiments using the coherent pumping.

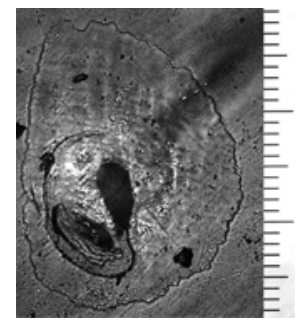

a

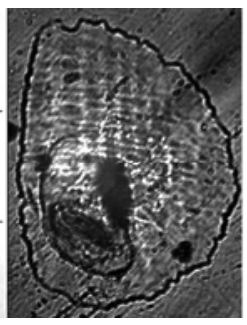

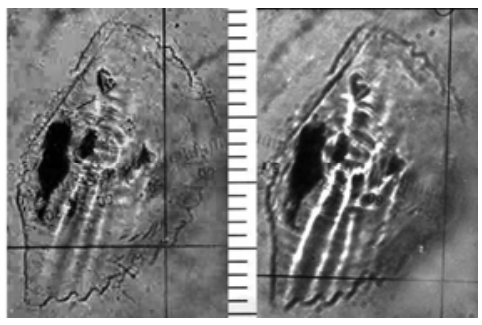

$\mathrm{b}$

Fig. 7. Structure changes in YAG plate in 2 different ccl shots $(a, b)$ : left - sample surface, right- 7-10 $\mu \mathrm{m}$ under the surface. Wavy lines on the photos are supposed to be caused by the hypersonic waves interference inside the sample, connected with the aberration (coma) of the focusing lens, $\mathrm{L}_{2}$. Scale $\sim 11,8 \mu \mathrm{m} /$ mark

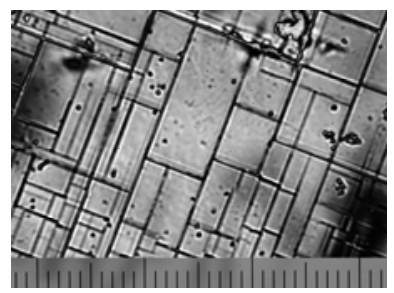

Fig. 8. Cracking at the LiF crystal surface. Scale $\sim 11,8 \mu \mathrm{m} /$ mark

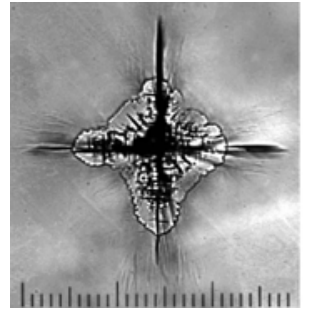

a

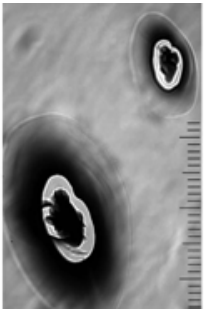

b

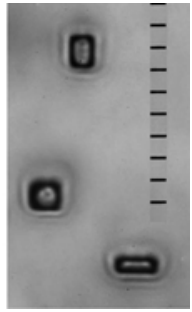

C

Fig. 9. Cross-like cracking having an additional plane formation with a sharp boundary inside the LiF crystal (a); ellipse-like cracking in the YAG crystal (b); a group of rectangular damages in the LiF crystal (c). Scale $\sim 11,8 \mu \mathrm{m} /$ mark

Structural changes in samples subjected to ccl pulses allow one to suppose the effect of hypersonic shock waves. Actually, a microphotograph of Fig.7a shows a region of the medium located at the depth of 7-10 $\mu \mathrm{m}$ under a YAG plate surface, where one can clearly see a quasi-periodic structure of stripes "imprinted" into an oval region with a sharp boundary. Figure $7 \mathrm{~b}$ demonstrates another region, where a similar structure with step 10-15 $\mu \mathrm{m}$ is shown. These structures happened to be visible thanks to the refractive index 
gradient. However, such structures could not be formed in a medium as a result of the interference of low-coherent $\mathrm{ccl}$ radiation. At the same time, these structures could be the result of interference of intensive hypersonic waves propagating at a small angle to each other in the region of SBS of pump radiation. There are some other experimental data, which support a hypothesis of origin of intensive hypersonic waves in the medium. For example, a sharp boundary outlining structural changes in an YAG crystal, Fig.7 is the evidence of matter displacement deep down caused by the motion of dislocations in a surface region probably due to the phase transition in the low-lying layers in the region of hypersonic waves propagation. Note, that no apparent disturbances of the sample surface were observed. It is seen that some elements at the surface inside the oval region are near the same as they are outside: the pre-radiation scratches across the region boundary being continued, Fig.7.

To interpret the phenomena observed (stimulated scattering, structural changes) we consider a physical picture of the ccl radiation interaction with the medium, and a possible mechanism of the hypersonic waves buildup. The pump radiation spectral width of $\approx 600 \AA$ permits one to suppose that a $\mathrm{ccl}$ pulse represents a sequence of ultra-short pulses (USP) of fs durations distributed chaotically within the $20 \div 30$ ns envelope of the pump pulse. Energy of a single USP is estimated as $0.1 \div 1 \mu \mathrm{J}$. Power density of separate ultra-short spikes could exceed $10^{10} \mathrm{~W} / \mathrm{cm}^{2}$. The interaction of ps and fs pulses with optical media was intensively studied, see for example (Nelson et al.,1982). The interaction of a single fs pulse $\left(10^{13} \mathrm{~W} / \mathrm{cm}^{2}\right.$ intensity) with transparent dielectrics (quartz, $\mathrm{LiF}, \mathrm{CaF}_{2}$, and others) produced generation of coherent optical phonons in the $\mathrm{THz}$ region leading to Raman scattering of probe pulses with ps delay (Gordienko et al., 2010; Merlin, 1997). The hypersonic waves (GHz region) were excited in liquids and solid-state media due to SBS on a space grating at the interference of two identical laser beams $\left(10^{10} \mathrm{~W} / \mathrm{cm}^{2}\right.$ intensity, ps durations) propagating at an angle to each other (Nelson et al., 1982; Robinson et al., 1984). In our conditions samples were irradiated by a succession of the chaotically distributed USP with a spectral range, which may be characterized by the central wavelength $\lambda_{p}$ and the wave vector $k_{p}=2 \pi / \lambda_{p}$.

The appearance of shock waves at low-coherent pumping and their absence at the coherent pumping may be due to different conditions of SBS of pump radiation for those two cases. As is known, the SBS of coherent pump is developed on density fluctuations, i.e. hypersonic waves of the thermal origin (Ritus, 1982; Starunov \& Fabelinsky, 1969). Aside from thermal fluctuations, there exist the stationary inhomogeneities in any solid-state medium, such as micro-inclusions, lattice inhomogeneities, dislocations. The stationary inhomogeneities may be the source of excitation of hypersonic waves and SBS. In fact, the interference of the incident and reflected pump radiation near inhomogeneity gives rise to a standing light wave with a spatial period $\lambda_{p} / 2 n_{p}$ where $n_{p}$ is the refractive index of the medium at $\lambda_{p}$. The standing wave amplitude is determined by the coefficient of the pump radiation reflection from an inhomogeneity. The standing wave causes modulation of the refractive index, which gives rise to a spatial structure with the period $\Lambda=\lambda_{p} / 2 n_{p}$, which is resonant to the incident pump radiation and can be considered as the "seed" of the hypersonic wave. This structure starts moving due to the absorption of the momentum of light at scattering of pump photons. However, the stationary inhomogeneities do not exert influence on the development of hypersonic waves at the quasi-continuous coherent pumping, because the constant generation of the perturbations near the inhomogeneity is a drawback to the development of hypersonic waves and SBS. 
At the pulsed low-coherence pumping, not only fluctuations but stationary inhomogeneities may be the source of SBS and hypersonic waves as well. Let an interaction of a single USP (a spike of pump) with the radiation reflected by an inhomogeneity produces a spatial structure with the period $\Lambda=\lambda_{p} / 2 n_{p}$ in a medium at the distance corresponding to the spike coherence length (for YAG, $n_{p} \approx 1.82, \Lambda \approx 0.25 \mu \mathrm{m}$ ). For USP with durations ranging 0.01-0.1 ps the coherence length makes 5-50 $\mu \mathrm{m}$. If the spike duration $t_{\mathrm{sp}}$ is less than half period of the hypersonic wave $t_{s p}<T / 2\left(T=\lambda_{p} / 2 n_{p} v_{s} \approx 50 \mathrm{ps}, v_{s} \approx 5 \times 10^{5} \mathrm{~cm} / \mathrm{s}\right.$-sound velocity in YAG) then after the spike end the periodical perturbations should propagate in the direction of pump and backward in the form of two travelling hypersonic waves, whose lifetime in the solid-state medium is $\geq 10^{-9} \mathrm{~s}$ for the $\mathrm{GHz}$ frequencies (Ritus, 1982; Starunov \& Fabelinsky, 1969). In this time interval, the hypersonic waves may fall under influence of many other USP pulses, which follow the first spike. The pump radiation affects the hypersonic waves, and as a result it is partially scattered with changing frequency due to the Doppler shift both to the Stokes and anti-Stokes sides. In the anti-Stokes case an atom moving towards pump is decelerated by absorbing momentum from a counter propagating pump photon. That stops eventually the counter-propagating hypersonic wave. At Stokes scattering, however, momentum from a co-propagating pump photon is transferred to an atom in the direction of its motion, supporting the hypersonic wave propagation. Therefore, only one from the two hypersonic waves, i.e. the wave moving along the pump direction is sustained. Herewith the scattered light wave at the Stokes frequency $\omega_{s}$ propagates towards the pump and stimulates emission (at frequency $\omega_{s}$ ) of the excited atoms, which absorbed a pump photon (at frequency $\omega_{p}$ ). The difference of the photons energy, $\Delta \omega=\omega_{p}-\omega_{s}$, determines the amplification of the hypersonic wave, co-propagating the pump. At the same time this wave is extended towards the pump source. In other words, the scattered light wave deflates energy from the pump to the co-propagating hypersonic wave and extends it toward the pump source by half of the coherence length of the USP. The following next pump spikes running over the hypersonic wave should extend it up to the sample input surface.

Because the spatial structure of the co-propagating hypersonic wave, which is resonant with pump, reflects effectively the incident radiation, the maximum photon energy and momentum are transferred to atoms in a "leading" edge of this structure facing the pump source. The energy is accumulated there as a result of a multiple run of USP pulses on a hypersonic wave, which stops near the surface unable to propagate further. Thus, the hypersonic wave amplitude rises. This provides jumps of pressure at the wave fronts, which make the sound velocity higher at the maxima and the leading fronts steeper. The hypersonic wave is transformed into a periodic shock wave due to this growing "anharmonicity". Thus concentration of acoustic energy near the input surface of the sample occurs.

A proposed model of shock waves formation at SBS of low-coherent ccl radiation makes it possible to interpret the experimental data on stimulated scattering and structure changes in the medium. A directed action of high-energy phonons causes structural changes near the sample surface, as shown on microphotographs of Figs.7-9. Release of acoustic energy and the rise of tension in a sub-surface layer may be evidenced by cracking of a LiF crystal surface, Fig.8. Other evidences may be the flat regions of structural changes, Figs.7, 9. The analysis of experimental data on lasing in the Yb-doped samples (see section 4-6) made it possible to estimate pressure at the shock wave fronts (tens of $\mathrm{GPa}$ ). The pressure above 10 $\mathrm{GPa}$ is higher than the pressure of phase transitions in some materials. One may assume that 
structural changes of YAG crystal with a sharp boundary, Fig.7, 9 are rather due to phase transitions in a medium.

It should be noted that propagation of a hypersonic wave produced at some inhomogeneity deep in the medium may be stopped inside the sample by a "barrier" in the form of a dislocation, a crack, and the like. The wave would be partially stopped by the barrier. Its acoustic energy is released in the sample near the side of the barrier. In the case of acoustic energy exceeding the crystal lattice deformation threshold or the phase transition threshold there occurs local splitting of medium, shift of the barrier's wall. In this way one can explain the observed structural changes in crystals occurring along cleavage planes, Fig.9. Figure 9a demonstrates a typical cross-like cracking of a LiF crystal having an additional plane formation with a sharp boundary. Figure $9 \mathrm{~b}$ shows an ellipse-like splitting in the YAG crystal. Sharp boundaries in a region of structural changes may be interpreted as phase transition boundaries in the medium. Figure 9c illustrates a group of rectangular damages, which seems to be due to the concentration of acoustic energy on defects (dislocation walls) inside the sample. The formation of tensions around damages, which seen in the form of blurred fringes, Fig.9c, is also the evidence of a considerable release of energy in that region. The comparison of mechanisms for hypersonic wave formation at SBS of the coherent and incoherent pump may be supplemented by the following considerations. The efficiency of the pump energy transfer into the acoustic energy proves to be higher in the case of the lowcoherence pumping. At similar pump energy the light-acoustic wave interaction proceeds more effectively when pumping by USP with higher intensities and at small distances (USP coherence lengths) as compared with the case of a high-coherence radiation. Rather large amplitude of light wave scattered on stationary inhomogeneities (as compared to fluctuation scattering) and high intensity of the low-coherence spikes (compared to average level of the pump intensity) should make the SBS threshold lower and stimulate formation of the intensive hypersonic waves. This explains the appearance of intensive hypersonic waves at low-coherence pumping. At the coherent pumping, the hypersonic wave seeded by a small-dimensional fluctuation of pressure, needs large distances for its development, and cannot "grow" to a shock wave. Note, that at the low-coherence pumping, the hypersonic wave may be caused even by a large-scale inhomogeneity, like a plane back surface of a sample. In that case, the produced hypersonic wave has a wide wave front. Even in a thin sample (1-2mm) this may cause structural changes of medium (phase transitions) comparable with dimensions of the pump spot.

Sharpening of the hypersonic wave fronts provides the appearance of high-frequency harmonics up to the optical phonons excitation. Moreover, as steepness of the shock waves increases, the momentum transferred to waves from successive USP pumping increases too, thus exciting oscillations of atoms (coherent phonons) with frequencies inversely proportional to the USP duration. The USP of the pump being scattered on coherent phonons (like probe pulses in works Gordienko et al., 2010; Merlin, 1997) give rise to the stimulated Stokes or anti-Stokes scattering. As optical phonons are concentrated on shockwave fronts, hence the stimulated scattering is linked to fronts, Fig.10. Due to a large shift of the Stokes wavelength $\left(\lambda_{s}\right)$, the SRS may be amplified only at the angle a to the pump: $\cos a=$ $n_{s} \lambda_{p} / n_{p} \lambda_{s}$, Fig.10. The anti-Stokes SRS is developed in the opposite direction. The respective directions and angles of scattering are illustrated in Fig.10. It should be noted that highintensity optical phonons excited at shockwave fronts stir up the molecules at amplitudes and frequencies close to the possible limit in the crystal lattice, and hence make easier structural changing of the medium, i.e. its phase transition to a more dense state. 


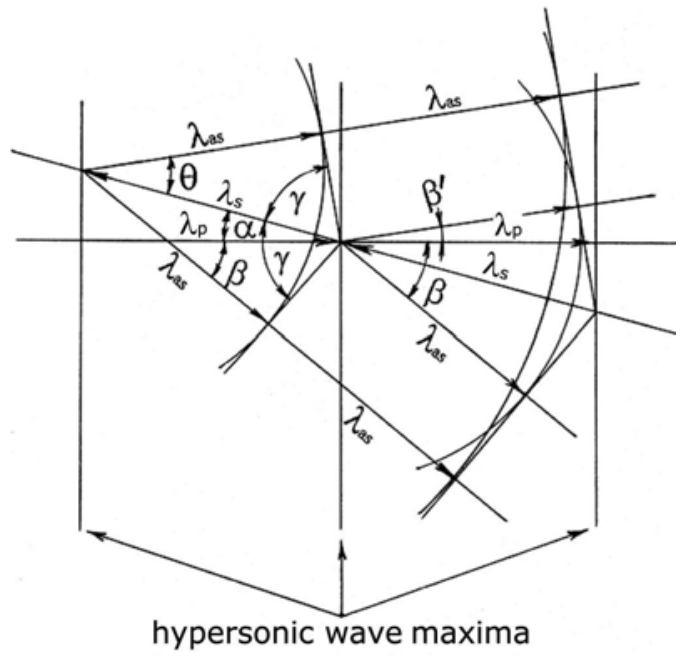

Fig. 10. Illustration to Stokes $\left(\lambda_{s}\right)$ and anti-Stokes $\left(\lambda_{a s}\right)$ scattering at hypersonic shock wave fronts: $a$ - angle between Stokes and pump waves; $\beta$, $\beta^{\prime}$ - angles between anti-Stokes and pump: $n_{s} \lambda_{p} / n_{p} \lambda_{s}=\cos a ; n_{s} \lambda_{a s} / n_{a s} \lambda_{s}=\sin \gamma=\cos \theta ; \theta-a=\beta^{\prime}$ and $\beta=90^{\circ}-(\gamma-a), \beta^{\prime}=90^{\circ}-(\gamma+a)$.

\section{Experiments on pumping Yb-doped media by LiF: $\mathrm{F}_{2}{ }^{+} \mathrm{ccl}$}

Experiments on pumping $\mathrm{Yb}$-doped media were performed using a setup of Fig. 3. Samples: plane - parallel 2-mm-thick $\mathrm{Yb}$ :YAG (20\% of Yb) or 3-mm Yb-doped phosphate glass $(10 \%$ of $\mathrm{Yb}$ ) plates were mounted in a resonator of length $\mathrm{L} \approx 20 \mathrm{~mm}$ formed by plane mirrors $\mathrm{M} 8$ and M9. Samples were pumped by pulses of focused ccl radiation through mirror M8 with the reflectance $\approx 100 \%$ at $\approx 1 \mu \mathrm{m}$ transmitting $80 \%-90 \%$ of pump radiation. The pump beam propagated close to the normal to the resonator mirrors. The reflectance of mirror M9 at $\approx 1$ $\mu \mathrm{m}$ was $30 \%$ (for $\mathrm{Yb}$ : YAG) or 70\% (for Yb: glass). The energy, spectrum, shape of Yb-laser pulses were registered by calorimeters, photodiodes, and STE-1 spectrograph. Experiments were performed at room temperature in the single-shot regime. In most experiments samples in the resonator were exposed to the ccl radiation of energy $60-70 \mathrm{~mJ}$. The focused ccl beam produced the radiation intensity distribution I (r) (r-radius of the excited region) in the subsurface layers of samples with the maximum at the pump beam axis. The minimal size $2 r$ of the focal spot on the sample surface was $\approx 250 \mu \mathrm{m}$. The ccl intensity was varied within $0.5-5 \mathrm{GW} \mathrm{cm}^{-2}$ by moving the lens. Lasing in a Yb :YAG crystal was observed at pump intensities exceeding $0.5 \mathrm{GW} \mathrm{cm}^{-2}$, Yb laser energy did not exceed $0.5 \mathrm{~mJ}$. Figures 11 and 12 show $\mathrm{Yb}$-lasing oscillograms recorded for one shot. The first of a series of laser pulses appeared during the pump pulse, while the next pulses appeared after the end of the pump and were delayed by tens and hundreds of ns, up to 1.2 microsecond ( $\mu \mathrm{s})$. Spectra were recorded by STE-1 on an IR film or on the image converter. The optical scheme of the STE-1 imaged (1:1) the vertical slit of $\approx 100 \mu \mathrm{m}$ width on the film. At uniform illumination of the slit by the monochromatic radiation of a spectral lamp the film registered separate narrow vertical lines, Fig.4b. In case of Yb-lasing we observed much more complex spectra, Figs 1114 . The lasing spectra were recorded within an angle of $\approx 10^{-2} \mathrm{rad}$, which was determined by 
the slit height and a distance $(\approx 1 \mathrm{~m})$ from the sample. Laser radiation going along the resonator axis was directed to the lower part of the slit. No focusing of radiation on the slit was performed. Figs 11, 12 demonstrate lines in the region of Yb transitions in YAG at 1.03 and $1.05 \mu \mathrm{m}$. At low pump intensities line lasing appeared first at $\approx 1.05 \mu \mathrm{m}$. As the pump intensity was increased, line lasing was observed simultaneously at 1.03 and $1.05 \mu \mathrm{m}$ (Fig. 11), and at the maximum pump it was observed at $1.03 \mu \mathrm{m}$, Fig.12. Fig.11 demonstrates a noticeable inclination of the spectral line at $1.03 \mu \mathrm{m}$ from the vertical direction. This means that the lasing wavelength in this line was varied along the slit height. Structure of smallscale $(50-200 \mu \mathrm{m})$ spots was observed in $1.05 \mu \mathrm{m}$ line, Fig.11. Along with narrowband lasing, broadband unstructured lasing was observed virtually in each pulse. Spectral bands up to $20 \mathrm{~nm}$ width were extended to the blue (up to $1.0 \mu \mathrm{m}$ ) and red (up to $1.06 \mu \mathrm{m}$ ) parts of the spectrum far beyond the regions near 1.03 and $1.05 \mu \mathrm{m}$ in which $\mathrm{Yb}$ :YAG lasing is usually observed. As pump was increased, the broadband spectra were shifted to the blue (Fig. 12).

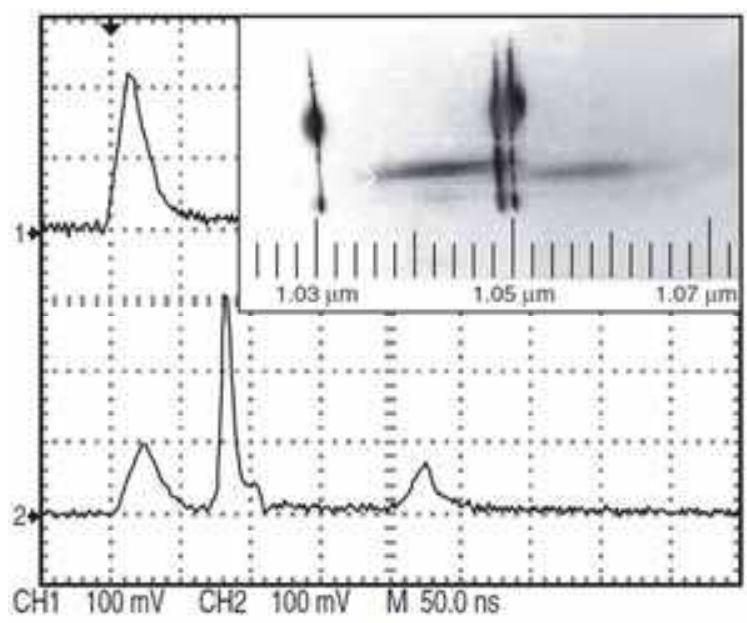

Fig. 11. Oscillograms of pump (1) and Yb:YAG laser (2) pulses, and the lasing spectrum in the $1.03-1.06-\mu \mathrm{m}$ region
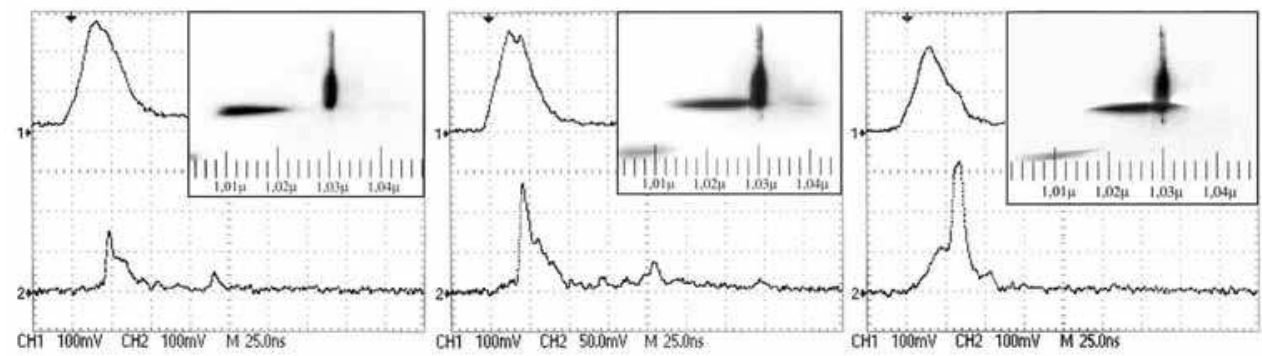

Fig. 12. Oscillograms of pump (1) and Yb:YAG laser (2) pulses and lasing spectra in 1.03 $1.06-\mu \mathrm{m}$ region for $3 \mathrm{ccl}$ pulses with energies 100, 125, and $150 \mathrm{~mJ}$ (from left to right) 


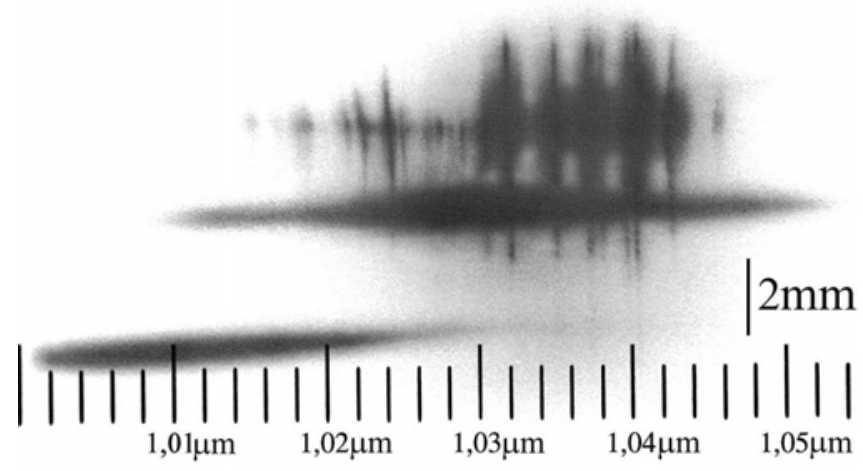

Fig. 13. Lasing spectra of $\mathrm{Yb}$ : glass in the $1.00-1.05-\mu \mathrm{m}$ region

Lasing in the $\mathrm{Yb}$ : glass (Figs 13,14) appeared at pump intensities 4-5 $\mathrm{GW} \mathrm{\textrm {cm } ^ { - 2 }}$ and was accompanied, as a rule, by the local damage of samples. The lasing energy did not exceed $0.1 \mathrm{~mJ}$. The oscillograms of the glass lasing (similar to the $\mathrm{Yb}$ :YAG lasing) exhibit several ns pulses delayed with respect to the pump, Fig.14. The spectrograms demonstrate line (in 1.02-1.05 $\mu \mathrm{m}$ region) and broadband $(1.00-1.06 \mu \mathrm{m})$ lasing spectra. Structures of small-scale spots are clearly visible in Yb:glass line spectra, Fig.14. The total spectrum of two broad bands of the Yb:glass emission exceeded $50 \mathrm{~nm}$, Fig. 13. The width of stripes of broadband lasing in vertical direction was about $1 \mathrm{~mm}$, Fig.13. The line and broadband lasing spectra both in glass and $\mathrm{Yb}$ :YAG were recorded from the slit parts located at different heights, Figs 11-14. Note, that spectra of $\mathrm{Yb}$ lasing were recorded when the slit was in the far-field diffraction zone with respect to the position of the radiation source. So, the observed narrow stripes and small spots of generation, Figs 11-14, indicated a high directivity of radiation. The angular divergence of radiation, estimated from the size of spots on the slit, did not exceed usually $10^{-3}-10^{-4} \mathrm{rad}$. At the same time, the angular divergence caused by diffraction from the lasing region with $\mathrm{r} \approx 100 \mu \mathrm{m}$ should be $\approx 10^{-2}$ rad. Thus, the angular directivity of Yb-lasing could exceed the diffraction limit by one- two orders of magnitude, i.e. the generation should occur in highly directed beams of small transverse size.

At pump intensities from 0.5 to $5 \mathrm{GWcm}^{-2}$ used in our experiments, we observed intense pulses of scattered ccl radiation in Yb-doped crystal and glass samples similar to observations of scattering (SBS and SRS) in non-doped samples described in the part 3. Consider the features of the inversion distribution in the Yb-doped medium upon SBS of pump radiation. The dynamic grating of hypersonic waves produced in samples near the surface played the role of an additional external mirror for the $\mathrm{ccl}$ and efficiently reflected incident radiation, preventing its propagation inside a crystal or glass sample. The penetration depth of hypersonic waves into the medium is $l \approx 1 / \eta,\left(\eta=10^{2-1} 0^{3} \mathrm{~cm}^{-1}\right.$ is the hypersonic waves attenuation coefficient) (Ritus, 1982; Starunov \& Fabelinsky, 1969). Thus, the excitation region of SBS (and SRS) in the medium was a surface layer of thickness $1<100$ $\mu \mathrm{m}$. The heat release caused by the dissipation of energy of hypersonic waves and optical phonons excited in the medium upon SRS of ccl radiation occurred in this layer. Inversion was also mainly produced in this thin layer. The maximum energy density stored in $\mathrm{Yb}$ :YAG containing $20 \%$ of $\mathrm{Yb}\left(\mathrm{N}_{0}=2.9 \times 10^{21} \mathrm{~cm}^{-3}\right)$ upon excitation of all $\mathrm{Yb}^{3+}$. ions to the ${ }^{2} \mathrm{~F}_{5 / 2}$ metastable level was estimated as $\approx 500 \mathrm{~J} \mathrm{~cm}^{-3}$. Even for such an extremely large energy in 
the volume of an $\mathrm{Yb}$ :YAG crystal cylinder of diameter $250 \mu \mathrm{m}$ and length $100 \mu \mathrm{m}$, the energy stored in inversion does not exceed, according to estimates, $2.5 \mathrm{~mJ}$, which corresponds to the low level of Yb-lasing energy in experiments. A part of the pump energy was absorbed by $\mathrm{Yb}$ ions beyond the region of SBS excitation, and the other part escaped from the sample. So, the population of $\mathrm{Yb}$ ions on the metastable level decreased over the sample length along the pump beam. Only a small fraction (estimated as $10 \%$ ) of the ccl output energy incident on samples was spent to excite ytterbium. A grater part (up to $70 \%$ ) of the ccl energy was transformed to stimulated scattering due to nonlinear interaction with the medium and was spent to form intense hypersonic waves and optical phonons accompanied by heat release in the medium. The thermal energy required for heating and melting of the above-mentioned $\mathrm{Yb}: \mathrm{YAG}$ crystal cylinder was estimated as 30 $\mathrm{mJ}$. At the same time, the thermal energy in the medium during the formation of inversion and generation and due to stimulated scattering did not exceed, according to estimates, $10 \mathrm{~mJ}$, i.e. it was insufficient for melting of the material. This suggests that structural changes in the surface layer of samples (Fig.7-9) could be produced by intense hypersonic waves appearing during the SBS of pump radiation. Thus, the Yb-doped medium during the SBS of pump proved to be divided into a layer of thickness $\approx 100 \mu \mathrm{m}$ with the very high inversion and the main part of the sample to which pump radiation hardly penetrated.

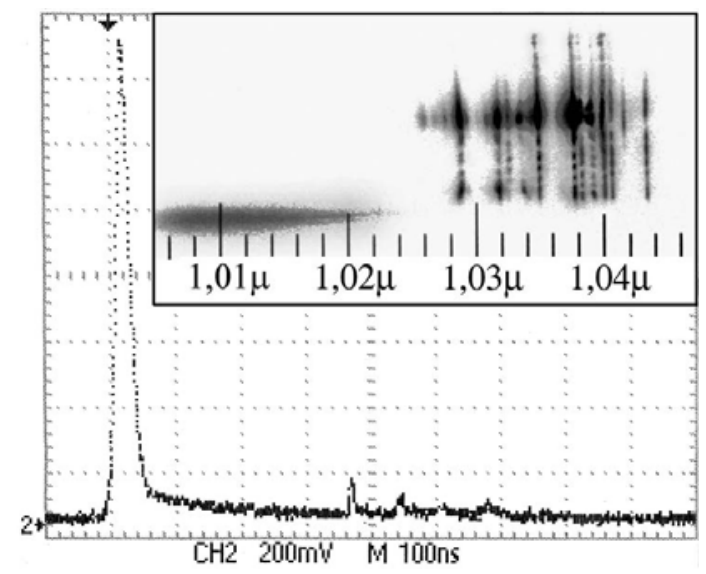

Fig. 14. Oscillograms of $\mathrm{Yb}$ : glass laser pulses and spectra in the $1.00-1.04-\mu \mathrm{m}$ region

Figures 11-14 demonstrate the integrated (per pulse) temporal pictures and broadband and narrowband lasing spectra of ytterbium. These two types of emission correspond to different $\mathrm{Yb}$-laser pulses appearing at different stages of the development of lasing. We can distinguish two such stages: the first one is the development of Yb-lasing during irradiation of a sample by the pump pulse, and the second one is Yb-lasing after the end of the pump pulse. At the first stage of duration $t_{1}=20-30 \mathrm{~ns}$, during the action of a ccl pulse onto the sample the generation could occur without a resonator, just due to the feedback at the dynamic hypersonic wave grating. The broadband unstructured spectra correspond to this stage. At the second stage of duration $t_{2}$ up to $1.2 \mu$ s there was generation in the $\mathrm{Yb}$ laser resonator which was characterized by line spectra, Figs. 11-14. 


\section{Features of $\mathrm{Yb}$ - lasing: broadband spectra, data interpretation}

We attribute broadband lasing in the crystal and glass to Yb-laser pulses appearing at the first stage. Consider the conditions of inversion formation and lasing at the first stage by using a model assuming the appearance of shock hypersonic waves in the medium during the SBS of pump radiation. Excitation of $\mathrm{Yb}$ ions to the $2 \mathrm{~F}_{5 / 2}$ metastable level and formation of hypersonic wave grating (Fig. 15) occurred simultaneously in a thin layer of the medium. The grating period $\Lambda$ is related to the pump wavelength $\lambda_{\mathrm{p}}$ and the refractive index of the medium $n_{p}$ by the expression $\Lambda=\lambda_{p} /\left(2 n_{p}\right)$ (Ritus, 1982; Starunov \& Fabelinsky, 1969). By assuming that $\lambda_{p} \approx 0.92 \mu \mathrm{m}$, we obtain for YAG $\Lambda \approx 0.25 \mu \mathrm{m}$. The period of the hypersonic wave is $T=\lambda_{p} /\left(2 n_{p} v_{s}\right)$, where $v_{s}$ is the sound speed. For the glass, we have $T \approx 70 \mathrm{ps}$, and for the YAG crystal, $T \approx 50$ ps. Intensive hypersonic waves strongly affected the spatial distribution of inversion. Indeed, conditions were produced for excitation of high-frequency vibrations of the medium (optical phonons) and SRS on the fronts of shock waves. The scattering parameters show that a broad phonon spectrum was excited upon SRS, including phonons with energies $\approx 1000 \mathrm{~cm}^{-1}$ near the high-frequency boundary of the phonon spectrum in the medium (Gorelik, 2007). The energy of such phonons is sufficient for the population of the Stark components of the ${ }^{2} \mathrm{~F}_{7 / 2}$ level lying above the ground level of $\mathrm{Yb}$ (Fig.1). The redistribution of the population of Stark levels occurs during several ps. Due to the phonon population on the fronts of shock waves, the inversion for transitions from the ${ }^{2} \mathrm{~F}_{5 / 2}$ level to the Stark components of the ${ }^{2} \mathrm{~F}_{7 / 2}$ level was decreased, preventing the development of lasing. At the same time, due to the rapid decrease of pressure and dynamic cooling of the medium, which occurred behind the fronts of shock waves (Fig. 15), the population of the Stark components of the ${ }^{2} \mathrm{~F}_{7 / 2}$ level was rapidly depleted, resulting in the inversion jump. As a result, a structure of thin $(<0.3 \mu \mathrm{m})$ periodical layers with the high inversion was established in the medium for a short time $\left(t_{i n v}<\mathrm{T}\right)$. During the propagation of a hypersonic wave, the inversion regions were displaced. After the time $\mathrm{T}$, the same inversion distribution over layers again reestablished. Thus, upon the SBS and SRS of pump radiation, amplification could appear at transitions between the ${ }^{2} \mathrm{~F}_{5 / 2}$ level and Stark components of the lower ${ }^{2} \mathrm{~F}_{7 / 2}$ level, including transitions in the short-wavelength region (< $1.03 \mu \mathrm{m})$, which are not observed usually in $\mathrm{Yb}$ - lasers.

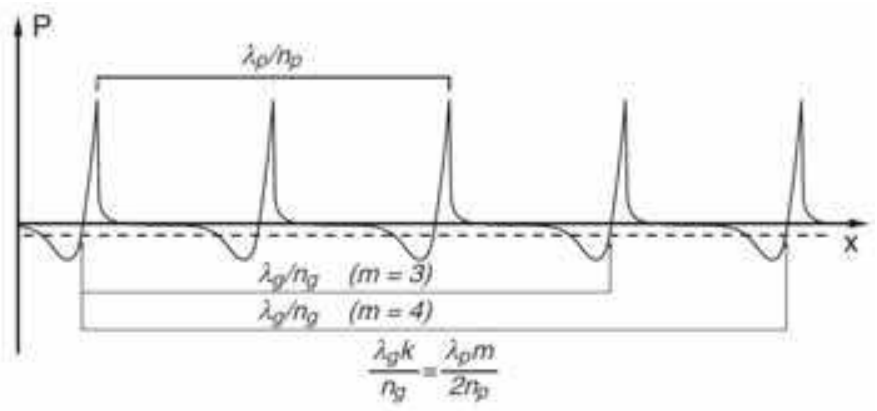

Fig. 15. Illustration to conditions for inversion formation and stimulated emission in the region of shock hypersonic waves in the active medium. The dependence of the pressure profile $\mathrm{P}$ in the hypersonic wave on the coordinate $x$ along the pump direction is shown. The dashed straight line indicates the pressure at which inversion is produced. The possible relations between the pump, $\lambda_{p}$ and laser, $\lambda_{g}$ wavelengths satisfying condition (1) are given 
The formation of inversion in the region of propagation of hypersonic waves produces the conditions for generation of short radiation pulses in the medium. Indeed, a distributed feedback (DFB) laser (Kogelnik \& Shank, 1971) appeared in fact in our experiments in the hypersonic wave grating region. Such a laser operates without an external resonator and can emit ps pulses (Bor \& Muller, 1986; Katarkevich et al, 1996; Kogelnik \& Shank, 1971). Unlike dye DFB lasers based on a photo-induced sinusoidal grating in the medium, the highcontrast DFB structure was formed under our conditions due to modulation of the medium parameters (refractive index, inversion) during the propagation of a sequence of intense hypersonic waves. The possibility of creating a DFB laser on a hypersonic wave grating was discussed already in pioneering paper (Kogelnik \& Shank, 1971). The length of the DFB structure in the region of SBS is $\approx 100 \mu \mathrm{m}$; in this case, the travel time $\mathrm{t}^{\prime}$ of a photon in the structure is much shorter than the round-trip transit time $\tau$ in the $\mathrm{Yb}$ - laser resonator $(\tau=$ $2 L / c \approx 100 \mathrm{ps}$ ). The possibility of lasing using the hypersonic wave grating without the resonator was confirmed by experiments. Lasing during the ccl pump pulse was observed both in samples mounted inside the resonator and in samples without the resonator, in particular, in samples mounted at an angle to the pump beam axis. The generation of ps pulses in dye DFB lasers on a stationary grating pumped by ns pulses occurs due to self-Qswitching mechanism: 'blowing away' of the gain grating by the structure emission itself (Bor \& Muller, 1986; Katarkevich et al, 1996). Unlike this, upon the SBS of ccl pump radiation, a DFB structure moving in the medium appears with period $\Lambda$ which can depend on the pump intensity (pressure in the medium). Under these conditions, the Q-switching in the DFB structure can be determined, apart from the mechanism considered above, by the rapid movement of the grating in the medium and by variations in its parameters as well. Pulses of ps duration, $t<t_{i n v}$ should be generated in our experiments successively in these periodic inversion layers during their movement. Besides, the change in $\Lambda$ should lead to scanning of the lasing spectral band over the gain profile in the medium. The temporal picture of $\mathrm{Yb}$ - lasing at the first stage (during pump) was not investigated in detail due to the lack of recording equipment with a proper time resolution. The shape and duration of $\mathrm{Yb}$-laser pulses were determined by the resolution of the oscilloscope (1- $4 \mathrm{~ns}$ ).

By considering the conditions for appearing of stimulated emission in the moving layer structure, we can interpret features of broadband lasing spectra of the glass and $\mathrm{Yb}: \mathrm{YAG}$ crystal (Figs 11-14). The $\mathrm{Yb}$ emission appears at wavelengths $\lambda_{g}>\lambda_{p}$. This emission is amplified along the normal to the layer structure when the path difference between the layers involved in lasing is equal to the quantity multiple of $\lambda_{g}$. This synchronism condition between hypersonic waves and lasing can be written in the form

$$
\frac{\lambda_{g} k}{n_{g}}=\frac{\lambda_{p} m}{2 n_{p}}
$$

Here, $n_{g}$ is the refractive index of the medium for $\lambda_{g}$; and $k$ and $m$ are integers. It is important that the refractive index depends on pressure $\mathrm{P}$ in the medium: $n_{g}, n_{p} \sim P$. Pressure depends on the intensity of hypersonic waves and the heat release in the medium upon pumping. The maxima of the spatial structure of the laser field are located in regions with a decreased density, behind the fronts of shock waves, Fig. 15 . Here $n_{g}$ can take values lower than the refractive index $n_{0}$ at the normal pressure. At the same time, the refractive index $n_{p}$ on the fronts of shock waves can be larger than at the normal pressure. Under these conditions for $n_{g}<n_{p}$, relation (1) is fulfilled for $k=1$ for small values of $m(m>2, m=3,4 \ldots$, see, Fig. 15). It 
follows from the relation $\Lambda=\lambda_{p} /\left(2 n_{p}\right)$ that, as the intensity of hypersonic waves and heat release increase (with increasing $P$ and $n_{p}$ ) at the fixed $\lambda_{p}$, the period $\Lambda$ of the grating decreases. This in turn leads to the blue shift of $\lambda_{g}$. Correspondingly, as $P$ is decreased, the wavelength $\lambda_{g}$ should shift to the red. Experiments confirm these conclusions. The shift of broadband spectra with changing the pump intensity is illustrated in Fig. 12. One can see that the spectrum of $\mathrm{Yb}$ :YAG shifts to the blue by $10 \mathrm{~nm}$ with increasing the ccl pulse energy from 100 to $150 \mathrm{~mJ}$. Note that the shift of the emission region of DFB lasers with changing of the pressure was observed in dye lasers (Bor \& Muller, 1986).

The inhomogeneous distribution of the pump with the maximum at the beam axis gave rise to the transverse gradient of pressure $P(r)$ in the region of propagation of hypersonic waves. Because of this gradient, for regions with different pump intensities (at the beam axis and its periphery) relation (1) is fulfilled for different values of $m$. These values can correspond to the regions of the broadband spectrum shifted in the wavelength scale and over the slit height, Figs 11-14. In the axial region with the maximum pressure $(r \approx 0$, the lower part of spectrograms), lasing was developed in the short-wavelength part of the spectrum. The spectra in regions with the lower pump $(r>0)$ are shifted to the red (Figs 11-14).

The regions of the broadband spectrum should be located on the wavelength scale in accordance with possible transitions in Yb: glass and Yb:YAG. According to the energy level diagram presented in Fig. 1, the emission of Yb:YAG in the spectral region under study (Figs $11,12)$ can be determined by transitions between the two lower components of the metastable ${ }^{2} \mathrm{~F}_{5 / 2}$ level with energies 10327 and $10624 \mathrm{~cm}^{-1}$ and three components of the ${ }^{2} \mathrm{~F}_{7 / 2}$ level with energies 565, 612, and $785 \mathrm{~cm}^{-1}$. In each spectral region, lasing in the DFB structure was built up in the active medium within the part of the gain line corresponding to the $\mathrm{Yb}$ transition which was resonant for the particular period of hypersonic waves. Thus, a large width of lasing spectra of Yb-doped materials (Figs 11 - 14) could be caused not only by the generation of short pulses in the DFB structure upon emission of thin layers of the active medium but, as explained above, by the shifts of $\lambda_{g}$ with changing the pump intensity as well. This is a picture of lasing in Yb-doped media in the region of the propagation of longitudinal hypersonic waves at SBS of pump radiation. Thus, the data on $\mathrm{Yb}$ broadband lasing confirm the concept of intense (shock) hypersonic wave formation at powerful lowcoherent pumping of the optical media.

The interpretation of data on the "sub-diffraction" angular divergence of Yb:YAG and Yb:glass generation had required the introduction of a new concept for the photon, which means its existence not in the form of a "traveling" wave, but with fixed positions of maxima, minima, and nodes along the photon propagation direction (Bykovsky, 2006; Bykovsky \& Senatsky, 2008a,b, 2010). It was assumed that the photon is a combination of the two pinpoint dipoles, which move jointly with light velocity and rotate with frequency $\omega$ in the opposite directions. The phase of the photon and its field are connected with the phase of this rotation (Bykovsky, 2006). Such an approach helps to describe the effects observed. The field produced by the photon motion along the coordinate $x$ is described by the function: $E=E_{0} \sin \left(k x-\varphi_{0}\right)$, where $k=2 \pi / \lambda$, and $\lambda$ is the distance, at which the photon changes its phase by $2 \pi$; $E_{0}$-the field amplitude, $\varphi_{0}$ - the initial phase. The new concept does not contradict to the properties of the well-known electromagnetic "traveling" wave: the traveling wave may be "constructed" from separate photons following each other along the same direction and having a continuous relative phase shift from 0 to $2 \pi$. If we assume that the excited atoms in a medium irradiate photons along the same direction and with the 
initial phases $+\pi / 2$ or $-\pi / 2$ only, and that emitted photons are correlated by the relation $\Delta x=c \Delta t$, where $\Delta x$ is the distance between the neighbor photons and $\Delta t$ - the interval between the moments of their emission, then such a combination of photons should form a traveling wave. This assumption follows from an equation describing a field of a neighbor photon irradiated from a point distanced by $\Delta x: E=E_{0} \sin \left[k(x \pm \Delta x) \pm \varphi_{0}\right]$. Taking into account that $k \Delta x=k c \Delta t=\omega \Delta t$ we obtain: $E=E_{0} \sin \left(k x \pm \omega \Delta t \pm \varphi_{0}\right)$ - the traveling wave relation. The traveling wave amplitude increases in proportion to the number of equal-phase photons. To provide the traveling wave formation it is necessary that the size of a homogeneous region with inversion, from which a large number of photons is spontaneously irradiated into $4 \pi$, exceeded the wavelength $\lambda$. Organization of photons in the form of the traveling wave makes it possible to deplete inversion with maximum efficiency, as it is taken off continuously from all points on the path of the wave propagating in the active medium. If the inversion is nonhomogeneous and structured with the period $\lambda / 2$, such a medium will emit photon in trains, whose maxima should spatially coincide with the inversion maxima. Photons having phases differing by $2 \pi n$ form a photon train described by the function: $E_{n}=E_{0} \sin \left(k x \pm 2 \pi n \pm \varphi_{0}\right)$. That should not be a traveling wave. 'The instant photograph' of the filed distribution in a photon train should represent the contrast periodic structure of antinodes and nodes and can noticeably differ from 'the instant photograph' of a traveling sinusoidal wave. However, the summary field produced by many photon trains, which are correlated by the relation $\Delta x=c \Delta t$, becomes "traveling". This "traveling" is like the Christmas-tree garland, whose running lights are due to the time shift of neighboring lamps burning moments.

Because the field produced by the photon is described by a harmonic function, the field superposition of the photon trains propagating at different angles and with different phases gives rise to the wave effects: interference and diffraction. The diffraction is connected with spatial confinement of the light wave front and caused by the interference of photons with different phases contained in the aperture of a beam. The intensity peaks are formed in the directions defined by the beam wave front profile, dimension and shape of a confined region, and the light wavelength. By the angular width of these peaks one may judge about the light beam divergence. The diffraction divergence of the traveling wave arises because the traveling wave restricted by an aperture contains within this aperture at the length of one spatial period the photons with different phases ranging from 0 to $2 \pi$. In general the phase dispersion of photons distributed in the interval $\Delta x$ along the beam direction can be estimated as $\Delta \varphi=2 \pi \Delta x / \lambda$. When the beam is limited by an aperture of diameter $d$, the direction to the first minimum of the far-field intensity distribution (half the angle a of the diffraction divergence) can be written in the form $\alpha \sim \lambda \Delta \varphi /(2 \pi d)$. In the case of a traveling plane monochromatic wave, the phase dispersion $\Delta \varphi$ in the beam can achieve $\pm \pi$. This gives the known estimation $\alpha \sim \lambda / d$ of the divergence angle for a beam limited by an aperture of diameter $d$. If the phase dispersion of photons is decreased, the diffraction divergence angle $\alpha$ decreases proportionally to $\Delta \varphi$. The group of such phased photons will form a beam with the sharp angular radiation pattern, which was observed in our experiments. Formation of beams with sub diffraction divergence occurs, apparently, in experiments on super-resolution microscopy, when the size of the fluorescent or light scattering object along the direction of beam does not exceed the light wavelength (Galbraith, C.\& Galbraith, J., 2011; Tychinskii, V., 2008).

Unlike a usual laser with the extended active medium in the resonator, in our experiments radiation was formed in a structure consisting of thin inversion layers. The regions with reduced pressure behind the front of shock waves, shown in Fig. 15, represent as if the 
'instant photograph' of the spatial arrangement of such a structure. The photons with the wavelength resonant with the structure, $\lambda_{g} / n_{g}=m \Lambda$, will occupy different spatial positions in the 'photograph' with respect to the layers of thickness $l_{i n v}$ « $\lambda_{g} / n_{g}$. And only a small part of the total number of photons in the 'resonator' of our DFB laser will coincide in position and phase with the inversion maxima in the medium and, therefore, will be amplified efficiently during the lifetime $t_{i n v}<T$ of the layers of the given spatial configuration, forming a beam on the basis of photon trains. During the movement of the inversion layers structure (following the movement of the hypersonic wave), new groups of phased photons will be formed, and their frequency will shift with changing the structure parameters. In such a way the model considered above explains the appearance of the sharp angular radiation pattern of the broadband lasing of ytterbium in the crystal and glass. In the case of a 'thick' sinusoidal grating, photons occupying different positions in space (which is equivalent to the large phase dispersion within the photon train) are amplified. This leads to the increase in the radiation divergence. Thus, when a periodic spatial grating of thin inversion layers is produced in the active medium, the high-directional stimulated radiation of an ensemble of excited atoms can be observed in the optical range.

\section{Features of ytterbium lasing: line spectra, data interpretation}

Line spectra observed in the crystal and glass relate to the second stage of $\mathrm{Yb}$-lasing. The second stage is the $\mathrm{Yb}$-lasing in the resonator after the end of pump due to inversion remained in the medium. Of special interest the details of $\mathrm{Yb}$ spectra are. Twisted, inclined and structured lines in spectra were observed in the region 1.03-1.05 $\mu \mathrm{m}$, Figs.11-14,16. Figures 11, 13, 14 showed an inclination of some lines to the short-wavelength side of the spectrum in respect to the vertical position of the slit. Nearly all line spectra showed quasiperiodic structures of small-scale $(50-200 \mu \mathrm{m})$ spots (Figs. 11, 14, 16). Twisted lines consisting of separate spots are shown at Fig.16. This picture indicates a wave-like change in the generation wavelength in separate lasing spots along the height of the slit.

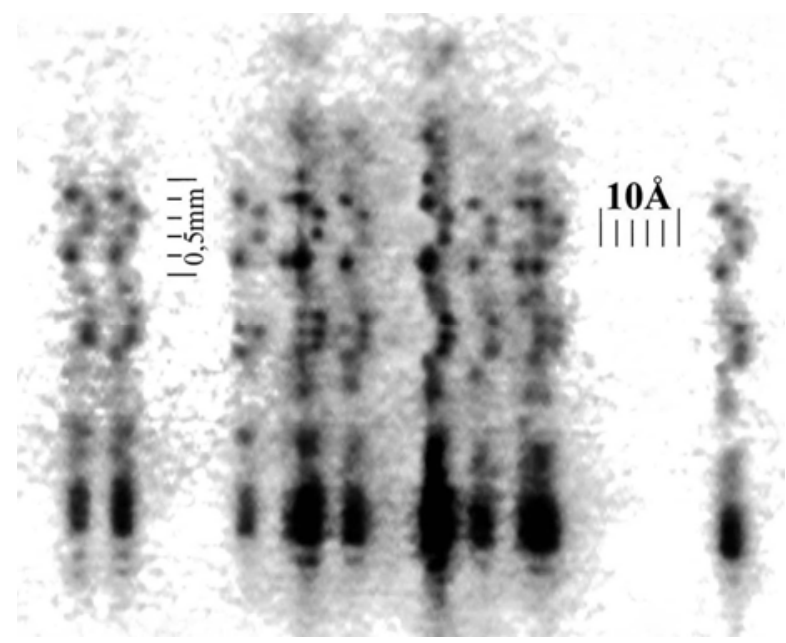

Fig. 16. Twisted lines with multiple spots of Yb:glass generation at several transitions near $1.04 \mu \mathrm{m}$; time delay from the pump pulse $\approx 50 \mathrm{~ns}$ 
Consider the conditions for the development of lasing in a resonator at the end of pump, under disappearance of longitudinal hypersonic waves and relaxation of the excitation region in the active medium. Lasing developed in the resonator in the presence of the region of strong optical inhomogeneity near the active element surface- a thin layer with the inhomogeneous inversion distribution and gradient of the refractive index, $n$ produced by pressure, temperature, and density gradients. To the rest part of the sample the pump penetrated weakly. The refractive index profile was formed during the action of the pump on a medium and it also changed after the end of pump during the relaxation of the excited region. This profile affected the development of Yb-lasing. An experimental study of a nonstationary refractive index profile (so-called "transient lens") arising under short laser pulse interaction with a medium is a rather complicated task. Here we discuss in a qualitative form processes that affected formation of the index profile, $n(r)$ assuming that pumping was symmetrical over the azimuth angle. Consider again a part of the medium in the form of a cylinder of $250 \mu \mathrm{m}$ diameter and $100 \mu \mathrm{m}$ length, which fits the size and configuration of the focal region at tight ccl focusing. The heat load occurred in such a cylinder during inversion formation, Yb-lasing, and mainly, due to pump radiation scattering. The heat load was estimated to be $<10 \mathrm{~mJ}$, which allows for the average thermal energy density in the cylinder, $<2 \times 10^{3} \mathrm{~J} / \mathrm{cm}^{3}$. Estimations for the average pressure, $P_{\mathrm{av}}$ and temperature, $T_{\mathrm{av}}$ just after the end of pump give $P_{\mathrm{av}} \approx 1 \mathrm{GPa}$ and $T_{\mathrm{av}} \approx 10^{3} \mathrm{~K}$. In actuality, due to the bell-shaped profile of the pump beam, $P(r)$ and $T(r)$ profiles with maxima exceeding averaged values arose in the medium. The maximum pressure near the axis of the excitation region at the moment near the end of pump pulse may be estimated by using the value $P_{\mathrm{pt}}$, at which a phase transition occurs. Structure modifications similar to phase transitions had been observed in Yb:YAG and Yb:glass samples near the axis, where hypersonic waves of maximum intensity propagated (section 3). We use data for glass $P_{\mathrm{pt}} \approx 10 \mathrm{GPa}, \delta n / \delta P=-0.13 \times 10^{-5} \mathrm{bar}^{-1}$, and $\delta n / \delta T=-6 \times 10^{-6} \mathrm{~K}^{-1}$ (Alcock \& Emmony, 2002; Koechner, 2006; Mak et al., 1990). At $P(0) \approx$ $P_{\mathrm{pt}}$ the drop of $n(r)$ along $r$ from axis to periphery of the cylinder due to the drop in pressure in the medium is $\Delta n\left(P_{\mathrm{pt}}\right)=\delta n / \delta P \times P_{\mathrm{pt}}=0.13$. Changes of $\Delta n$ due to temperature gradients are significantly less. For example, at $\Delta T=10^{3} \mathrm{~K}, \Delta n(T)=\delta n / \delta T \times T=-6 \times 10^{-3}$. For YAG $\delta n / \delta T \approx 8 \times 10^{-6} \mathrm{~K}^{-1}$ (Koechner, 2006; Mak et al., 1990) and $\Delta n(T) \approx 8 \times 10^{-3}$.Thus, the main contribution to the $n(r)$ profile was made just by pressure. The $n(r)$ profile defines a sign, optical power and aberrations of a "transient" lens in the medium. Optical power of the lens after the end of pump can be estimated at the assumption that the index had been changed by $\Delta n \approx 0.1$ at $\Delta r \approx 150 \mu \mathrm{m}$. That gradient corresponds to appearance in the medium of a positive lens with diameter $2 r \approx 300 \mu \mathrm{m}$ and $f \approx 1 \mathrm{~cm}$. A simplified scheme for the case of lasing in the resonator with a "transient" lens is given in Fig. 17.

The evolution of profiles $P(r)$ and $n(r)$ after pumping was determined by unloading of the high-pressure region in the focal volume. A problem of unloading of a small cylindrical or spherical region in a solid-state optical medium, where the energy had been stored after ns laser pulse irradiation, had been considered in several studies (Bullough \& Gilman, 1966; Conners \& Thompson, 1966; Sharma \& Rieckhoff, 1970). A typical process of relaxation is the propagation of an elastic dilatational wave across the medium. The time of traveling, $t_{t r}$ of an elastic wave with the sound velocity across the excitation region can be evaluated by $t_{t r}$ $=r / v_{s}$. For the glass, at $r_{p}=250 \mu \mathrm{m}$ and $v_{s}=4.5 \times 10^{5} \mathrm{~cm} / \mathrm{s}, t_{t r} \approx 50 \mathrm{~ns}$. The propagation of the dilatational wave along radial direction outward the center of the pumped region had led to the pressure profile deformation. With the drop of pressure at the axis, the gradient $d n / d r$ 
decreased accordingly. So, in the course of relaxation there occurred smoothing of profiles $P(r)$ and $n(r)$. It should be noted that profile $n(r)$ could include a small-scale modulation as well (imitated at Fig.17). The sources for such modulation might be pressure and density perturbations that had been arising in a medium at propagation of hypersonic waves during and after pumping. Note, that typical time of thermal relaxation of the medium in a cylindrical region with radius $r_{p}$ is $\tau=r_{p} 2 / 4 \mu » t_{\mathrm{tr}}$. Here $r_{p}$ is the radius of the pumped region and $\mu$ is the coefficient of the temperature diffusivity. For Yb:YAG at $r_{p}=250 \mu \mathrm{m}, \mu=0.046$ $\mathrm{cm}^{2} \mathrm{~s}^{-1}$ (Koechner, 2006 ), $\tau \approx 3$ ms. Hence, the time of thermal relaxation of medium is several orders higher than dynamic unloading. That is, roughly, a picture of formation and evolution of the refractive index profile in the medium in the region of ccl focusing defined by a non-uniform heat load and dynamics of the pressure profile during pumping and under the medium relaxation. So, a sequence of acoustic waves diverging in radial direction outward the focal region should affect the build-up of Yb-lasing in the resonator.

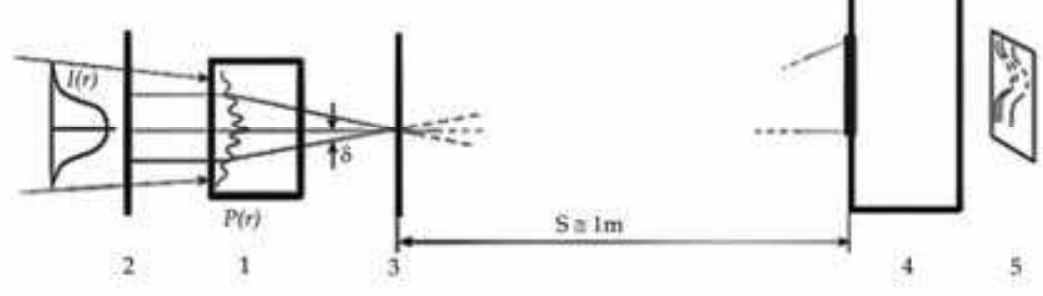

Fig. 17. Scheme of the experiment on excitation of $\mathrm{Yb}$-doped medium in the resonator by focused ccl radiation: 1 - active element; 2, 3 - mirrors; 4 - spectrograph STE-1 located at distance $S \approx 1 \mathrm{~m}$ from the resonator; 5 - a picture imitating spectra registered; $\delta$-angle between a generation trajectory and resonator axis. The dimensions of the resonator with active element are exaggerated relative to the scale of the scheme. Profiles of pump beam intensity, $I(r)$ and pressure in the medium, $P(r)$ are out of scale as well

The interpretation of data on the Yb-lasing in the resonator has required also the introduction of a new concept for the photon electromagnetic field distribution in space, considered in section 5. This new approach allows the existence of light beams of an aperture $d \approx \lambda$ and with a "sub-diffraction" angle of divergence. Within the framework of this new concept the picture of $\mathrm{Yb}$ lasing in the resonator looks as follows. The location of line spectra in the upper part of the slit evidences that $\mathrm{Yb}$ radiation emergent from the resonator was deflected from the axis, i.e., off-axis oscillations were built up in the resonator. For the arrangement of mirrors and active element given in our experiment off-axis lasing could be developed over trajectories with reflections at angles $\delta_{1}, \delta_{2} \ldots$ at the mirror (3), Fig. 17. Such trajectories located at distances $r_{\mathrm{pt}}<r<r_{p}$ from the axis, should contain curvilinear segments in the area of the optical inhomogeneity. We suppose that beams with aperture of several $\lambda$ (small generation channels) constituted lasing along these off-axis trajectories. At the high value of $d n / d r$ and the very high level of inversion, in each of the channels the selection of emitted photons (in accordance with their phases) took place in such a way that only a group of photons being approximately in the same phase $\varphi_{0}$ overcame the generation threshold. The photons different by phase from $\varphi_{0}$ were scattered due to diffraction and left the channel aperture. In the given channel with a coordinate $r$ a group of photons of the 
same phase made several round trips in the resonator during the time $t^{\prime}$, and a beam of the high directivity with the generation wavelength $\lambda_{\mathrm{g}}$ corresponding to one of the longitudinal modes was formed. So, generation in different channels over the pumped area developed at their own frequencies, and this radiation, reaching the slit at different points gave us an integrated picture of lasing beams and the spectrum in the form of lines composed of small spots, which dimensions characterized beam divergence in each of the channels.

For $\mathrm{Yb}$-doped plates placed in the middle of the resonator with $L=20 \mathrm{~mm}$ and for trajectories located in plates at $r<500 \mu \mathrm{m}$, angles $\delta$ should lie inside the spectrograph registration angle $\approx 10^{-2}$ rad. Beams of generation left resonator at angles $\delta$ and reached the slit. The greater was the distance $r$ between the generation trajectory inside the sample and the resonator axis, the greater was the shift of the corresponding generation beam spot upward in vertical direction on the slit, Fig. 17. So, under the propagation of beams of highdirectivity from resonator at angles $\delta$, one, actually, observed at the slit a projection (a magnified "image") of a spatial distribution of generation channels in the active medium over the radial coordinate. Since data on the $\mathrm{Yb}$ emission intensity distribution over the azimuth angle were absent, one can speak only about partial mapping on the slit of the spatial distribution of generation channels in the lasing area. The magnification coefficient $k$ of such an image may be estimated from comparison of dimensions of the possible generation area $(r \leq 500 \mu \mathrm{m})$ and the height of line spectra at the slit $(\approx 1 \mathrm{~cm}), k \geq 20$. With account of $k$ factor, to observed structures of small-scale spots in line spectra should correspond sources of $\mathrm{Yb}$ emission with dimensions of several wavelengths only.

Structures of multiple spots in Yb:YAG and Yb:glass lines (Figs. 11, 14, 16) may find explanation when one takes into consideration the specific spatial configuration of the field of thermo-elastic stresses in the area of ns laser pulse focusing into the medium. The quasiperiodic, alternating in sign, oscillating character of the amplitude of the tangential component of stress in dependence on the radial coordinate inside a small spherical region of a solid-state optical medium at ns laser pulse focusing was ascertained (Conners \& Thompson, 1966; Sharma \& Rieckhoff, 1970). In spherical ring zones round the centre of the focal region the tangential tensile stresses in the medium are consequently replaced by compressing stresses, and then, again, by the tensile ones, etc. The spatial period of such oscillations calculated for the case of $\approx 70 \mathrm{~mJ}$ laser pulse focusing into the glass constituted 30 $\mu \mathrm{m}$ (Sharma \& Rieckhoff, 1970). In conditions of our experiment one can expect the occurrence of oscillating (in space and time) profiles of thermo-elastic stresses in $\mathrm{Yb}$-doped media within the area of ccl radiation focusing as well. Alternating in sign stresses should result in a small-scale modulation at the profile $n(r)$, Fig.17. The oscillation of the $n(r)$ profile should stimulate the $\mathrm{Yb}$ generation in ring zones, which may fall into separate generation channels. The structures of bends consisting of generation spots, Figs.16 may be considered as a kind of an "image" of the distribution over the radial coordinate of tangential stress peaks in the focal region projected with a magnification on the slit. It is possible to say that such images were taken by the high-speed photography method. The "illumination" for this high-speed photography came from the $\mathrm{Yb}$ laser pulse itself. The "exposure" time of a single frame corresponded to the duration of the generation pulse. Time delay between the pump pulse and the "shooting" moments makes $\approx 50$ ns (Figs. 16) and over 300 ns (Fig.14). Splitting of a single bend into several generation spots with wavelength shifts (Fig.16) is in agreement with the model of generation channels. The shift of a spot to the long-wavelength side corresponds to increase of pressure (stresses) in the medium, and the shift to the short- 
wavelength - to decrease of pressure. In the course of medium relaxation, stress amplitudes and, correspondingly, spot wavelength shifts should have been reduced. At $\approx 50 \mathrm{~ns}$ delay (twisted lines, Fig.16) spots have noticeable wavelength shifts which correspond to high stress amplitudes. Structures at moments over $300 \mathrm{~ns}$ after pump (Fig.14) correspond to a smoothed picture of $n(r)$ profile. Note, that structures recorded with such big delays indicate the continued acoustic "ringing" in the medium. Estimated from Fig. 11, 14, 16 (at $k=20$ ) period of stress spatial oscillations in YAG and glass varies from 15 to $40 \mu \mathrm{m}$ and is in a qualitative agreement with the calculations (Sharma \& Rieckhoff, 1970). Acoustic vibrations frequencies corresponding to these values constitute $10^{8}-10^{9} \mathrm{~Hz}$. Attenuation of phonons at these frequencies at a room temperature for YAG is smaller than $0.1 \mathrm{db} / \mu \mathrm{s}$ and for glass about $10 \mathrm{~cm}^{-1}$ (Dutoit, 1974; Zhu et al., 1991). These data confirm that "ringing" of the unloading medium in the focal region in YAG and glass may continue over several $\mu$ s.

The Yb:YAG line spectra near 1,03 $\mu$ m were registered usually with $10 \div 50$ ns delays after the end of ccl pulse and even together with the trailing edge of the ccl pulse. Observations of line spectra near $1,03 \mu \mathrm{m}$, emitted soon after the pump pulse, like Fig.11, reveal noticeable inclination of spectral lines. This bending means that the wavelength of lasing at the same longitudinal mode (the number of nodes is preserved) changes from the centre to periphery of the excited region in the active medium. This corresponds to the development of lasing in some sites of the medium with a pressure (refractive index) gradient from the beam centre to its periphery. It can be easily shown that $\lambda_{g}$ shifts to the blue if the refractive index gradient decreases from the beam axis to its periphery, Fig. 11. The pressure drop $\Delta P$ in the medium after the end of pump can be estimated from this line spectral shift. The frequency $\omega_{q}$ of the longitudinal mode of the resonator with the number of wavelengths $\lambda_{q}$ over the resonator length $2 L$ equal to $q$ is described by the expression

$$
\omega_{q}=\frac{\pi c q}{(L-\Delta l) n_{0}+\Delta l \cdot n(r)}
$$

where $\Delta l$ is the longitudinal size of the optical inhomogeneity of radius $r ; \mathrm{n}_{0}$ is the averaged refractive index outside the nonlinearity region; and $n(r)$ is the refractive index in the nonlinear region. It follows from (2) that the change in the mode frequency $\omega_{q}$ during the displacement along the radius from $r_{1}$ to $r_{2}$ is

$$
\Delta \omega_{q}=\frac{\pi c q \Delta l\left[n\left(r_{1}\right)-n\left(r_{2}\right)\right]}{\left[(L-\Delta l) n_{0}+\Delta l \cdot n\left(r_{1}\right)\right]\left[(L-\Delta l) n_{0}+\Delta l \cdot n\left(r_{2}\right)\right]}
$$

By substituting the expression for $q=\frac{2\left[(L-\Delta l) n_{0}+\Delta l \cdot n(r)\right]}{\lambda_{q}(r)}$ into (3) and assuming that $\Delta \ln (r)<L n_{0}$, we obtain the dependence of the change in the refractive index, $\Delta n$ on $r$

$$
\Delta n=\frac{\Delta \lambda_{q}(r) L n_{0}}{\lambda_{q} \Delta l}
$$

Here, $\Delta \lambda_{q}(r)$ is the wavelength shift along the radius. For $\Delta \lambda_{q}(r) \approx 1.4 \times 10^{-7} \mathrm{~cm}, \lambda_{q} \approx 10^{-4} \mathrm{~cm}, \mathrm{~L}$ $\approx 2 \mathrm{~cm}, n_{0} \approx 1$, and $\Delta l \approx 10^{-2} \mathrm{~cm}$, the change in the index is $\Delta n \approx 0.28$. By assuming that the change in the index is produced only by the change in pressure along the radius and using 
the value of $d n / d P$ for glass (Alcock \& Emmony, 2002), we obtain $\Delta P \approx 30 \mathrm{GPa}$. So, this estimates the pressure, which arises at the axis of the focal region after the end of pump.

Similar estimations are possible for the pressure jumps, which arise during the propagation of dilatational waves outward the center of the focal region. Knowing a value of the wavelength shift in a bend at Fig.16, $\Delta \lambda_{q}(r)$, one can estimate changes of the index, $\Delta n$ and pressure, $\Delta P$ in the medium, using the expression (4). As seen from Fig.16, for glass at $\Delta \lambda_{q}(r)$ $\approx 0.3 \times 10^{-7} \mathrm{~cm}, \lambda_{q} \approx 10^{-4} \mathrm{~cm}, L \approx 2 \mathrm{~cm}, n_{0} \approx 1, \Delta l \approx 10^{-2} \mathrm{~cm}, \Delta n \approx 0.05$, and $\Delta P \approx 5 \mathrm{GPa}$. The estimated $\Delta P$ considerably exceeds the glass fracture strength $<0.1 \mathrm{GPa}$ (Sharma \& Rieckhoff, 1970), which was measured usually for applied static load. The role of tensile stresses in laser damage of transparent dielectrics was discussed in many publications (Koldunov et al., 2002; Sharma \& Rieckhoff, 1970; Strekalov, 2000). It was considered that due to oscillating character of the stress amplitude in the focal area there must be observed laser damage of the medium in the form of periodically spaced spherical rings (Sharma \& Rieckhoff, 1970; Strekalov, 2000). It is known that material strength sharply grows under pulsed load as compared to the static load. Under the high-speed deformation (in the ns range), strength of material becomes comparable to the theoretical limit-tens GPa (Kanel et al., 2007). So a fast periodic change of the stress sign in the medium should decelerate the development of material destruction in the form of rings. This is the reason why the laser damage in the form of multiple rings usually was not observed in many experiments when ns pulses of laser radiation were focused into the volume of transparent dielectrics. The damage in the form of rings was not observed in our experiments as well. One of the few observations of the multiple ring damage (Martinelli, 1966) relates to the case when glass samples were exposed to focused free-running laser radiation. Anyway, according to calculations (Sharma \& Rieckhoff, 1970) oscillating profile of thermo-elastic stresses should occur in the region of ns laser pulse focusing. The presented material provides experimental data which confirm in the quality form the calculated (Sharma \& Rieckhoff, 1970) picture of thermo-elastic stresses distribution and elastic wave propagation across the medium.

\section{Conclusion}

The study of the interaction of powerful ns pulses of low-coherence radiation of the LiF: $\mathrm{F}_{2}{ }^{+}$ color center laser (ccl) with optical materials (Yb:YAG, glass, et al.) was carried out. Efficient SBS of low-coherence pump, accompanied by SRS and formation of hypersonic waves reaching the intensity of shock waves were found. A physical model of excitation of SBS and hypersonic waves at scattering of ultrashort pulses of low-coherence pump at stationary inhomogeneities in optical materials is presented. It is shown that ns laser pulse, whose duration is much higher than its inverse spectral width, causes SBS much more efficient than a pulse of high coherence with the same duration and energy. Unlike SBS of a coherent radiation caused by a pressure fluctuation, scattering of low-coherence pump may be caused by any stationary inhomogeneities in a medium: cracks, dislocations, microinclusions, or just by a plane back surface of a sample. An effective energy contribution of light pulses into hypersonic waves on a small coherence length near the input surface of a sample leads to their transformation into a periodic succession of high-pressure shock waves, which results in structure changes of a crystal lattice (phase transition) in that region. The appearance of structural changes in optical materials that are specific to the interaction of powerful pulses of low coherence radiation with matter was found. The mechanisms of structural changes based on the action of intense hypersonic waves were considered. 
Nanosecond pulses of $\mathrm{Yb}$ lasing in the region 1.00-1.06 $\mu \mathrm{m}$ with the spectral width up to 20 $\mathrm{nm}$ in $\mathrm{Yb}$ :YAG and $50 \mathrm{~nm}$ in the $\mathrm{Yb}$ : glass samples were observed. The divergence of the broadband laser radiation $\left(10^{-3}-10^{-4} \mathrm{rad}\right)$ was one or two orders of magnitude smaller than the diffraction limit respectively to the source of $\mathrm{Yb}$ radiation in a sample. The mechanism of generation of broadband laser pulses of short duration and high directivity in the spatial structure of thin layers with inversion produced in the region of the propagation of intense hypersonic waves in the medium is discussed. The interpretation of experimental data is based on a new concept of the spatial distribution of the electromagnetic field of a photon not in the form of a "traveling" wave but with the field structures located in fixed positions along the photon propagation direction. The new approach allows the existence of light beams of an aperture $d \approx \lambda$ and with a "sub-diffraction" angle of divergence. Such beams must consist of groups of phase-synchronized photons with a small phase difference distanced by an interval $\approx \lambda$. This synchronized group is no longer a "traveling" wave, its angle of divergence is defined by the phase difference of photons in a group.

Spectral lines in 1.03-1.05 $\mu \mathrm{m}$ region structured by $50-200 \mu \mathrm{m}$ spots as well as lines with inclinations were found at $\mathrm{Yb}$ lasing in a resonator. Structures of multiple spots in spectra reflect the specific spatial configuration of the field of thermo-elastic stresses in the unloading region of ccl pulse focusing after the end of the pump. Inclinations of spectral lines reflect the pressure gradient from the center to the periphery of the region of $\mathrm{ccl}$ focusing. Basing on inclinations of spectral lines the pressure in the region of shock hypersonic wave propagation was estimated. Estimations show that for some of the studied media the pressure values may exceed the phase transition threshold.

\section{Acknowledgment}

Authors thank O. Yaremchuk for the help in preparing this article for publication.

\section{References}

Alcock, R. \& Emmony, D. (2002). Sensitivity of reflection transducers J. Appl. Phys., Vol. 92, No.3 (August 2002), pp. 1630-1643, ISSN 0021-8979.

Basiev, T. et al. (1982). Solid-state tunable lasers based on color centers in ionic crystals Bulletin of the Academy of Sciences of the USSR, ser. phys., Vol. 46, No.8, pp.145-154.

Basiev, T.T, Bykovsky, N.E, Konyushkin, V.A \& Senatsky, Yu.V. (2004). Use of a LiF colour centre laser for pumping an Yb:YAG active medium. Quantum. Electron. Vol.34, No.12, pp. 1138-1142, ISSN 0368-7147.

Bor, Z. \& Muller, A. (1986). Picosecond distributed feedback dye lasers IEEE J. Quantum Electron., 22 (8), 1524-1533. ISSN: 0018-9197.

Bullough, R. \& Gilman, J.J. (1966). Elastic Explosions in Solids Caused by Radiation. J. Appl. Phys. Vol.37, pp.2283-2288, ISSN 0021-8979.

Bykovsky, N. \& Senatsky, Yu. (2008a). Broadband collimated generation in YAG:Yb crystal and ytterbium glass under LiF:F2+color center laser pumping. Laser Phys. Lett. Vol.5, Iss. 9. pp. 664-670, ISSN 1612-2011.

Bykovsky, N.\& Senatsky, Yu. (2008b). Spectra, temporal structure, and angular directivity of laser radiation of a Yb:YAG crystal and ytterbium glass pumped by low-coherence radiation from a F2+:LiF colour centre laser. Quantum Electron. Vol.38, No.9, pp. 813-822, ISSN 0368-7147. 
Bykovsky, N.E. \& Senatsky, Yu.V. (2010). Twisted lines and small-scale structures in generation spectra of $\mathrm{Yb}$-doped media excited by focused radiation of LiF:F 2+ color center laser. Laser Physics Vol.20, No.2, pp. 478-486, ISSN 1054-660X.

Bykovsky, N.E. (2005). Preprint FIAN No. 16. (http:/ / ellphi.lebedev.ru/12/pdf16/pdf).

Bykovsky, N.E. (2006). Preprint FIAN No. 36. (http:/ / ellphi.lebedev.ru/17/pdf36/pdf).

Conners, G. \& Thompson, R. (1966). A Continuum Mechanical Model for Laser-Induced Fracture in Transparent Media. J. Appl. Phys. Vol.37, pp.3434-3441, ISSN 0021-8979.

Dutoit, M. (1974). Microwave phonon attenuation in yttrium aluminum garnet and gadolinium gallium garnet. J. Appl. Phys. Vol.45, pp.2836-2841, ISSN 0021-8979.

Galbraith, C. \& Galbraith, J. (2011). Super-resolution microscopy at a glance Journal of Cell Science Vol.124, №10, pp.1607-1611 ISSN: 0021-9533

Gordienko,V., Mikheev P. \& Potemkin F. (2010). Generation of coherent terahertz phonons by sharp focusing of a femtosecond laser beam in the bulk of crystalline insulators in a regime of plasma formation.JETP Letters, Vol.92, No.8, pp.502-506, ISSN 00213640 .

Gorelik, V. (2007). Optics of globular photonic crystals. QuantumElectron, Vol.37(5), pp. 409432, ISSN 0368-7147.

Kanel, G., Fortov, V. \& Razorenov, S. (2007). Shock waves in condensed-state physics. Phys. Usp. Vol.50, pp.771-791, ISSN: 0038-5670.

Katarkevich, V., Kurstak V., Rubinov, A., \& Efendiev T. (1996). Kinetics of the operation of a distributed-feedback dye laser with nanosecond excitation. Quantum Electron.,Vol. 26, pp.1061- 1064, ISSN 0368-7147.

Koechner, W. (2006). Solid State Laser Engineering, 6th ed., Springer, Berlin.

Kogelnik, H., \& Shank, C. (1971). Stimulated emission in a periodic structure. Appl. Phys. Lett., Vol.18 (4), pp.152-155, ISSN 0003-6951.

Koldunov, M., Manenkov, A. \& Pokotilo, I. (2002). Mechanical damage in transparent solids caused by laser pulses of different durations. Quantum Electron., Vol.32. No.4, pp.335-340, ISSN 0368-7147.

Krupke W.F. (2000). Ytterbium solid-state lasers. The first decade IEEE J. Sel. Top. Quantum Electron., Vol.6 (6), pp.1287 - 1296, ISSN1077-260X.

Mak, A \& Soms, L. (1990). Nd:Glass Lasers, Nauka, Moscow.

Manenkov, A. \& Prokhorov, A. (1986). Laser-induced damage in solids. Sov. Phys. Usp., Vol.148, pp.104-122. ISSN: 0038-5670.

Martinelli, J. (1996). Laser-Induced Damage Thresholds for Various Glasses. J. Appl. Phys., Vol.37, pp.1939-1941, ISSN 0021-8979.

Merlin R. (1997). Generating coherent THz phonons with light pulses. Solid State Commun., Vol.102, pp.207-220, ISSN 0038-1098.

Nelson K.A., Miller R.J.D. \& Fayer M.D., (1982). Optical generation of tunable ultrasonic waves. J. Appl. Phys., Vol.53, pp.1144-1149, ISSN 0021-8979.

Polyakova A.L. (1968). Elastic Nonlinearity in Stimulated Mandel'shtam-Brillouin Scattering. JETP Letters, Vol. 7, Iss. 2, pp. 57-59, ISSN: 0021-3640.

Polyakova, A. (1966). Nonlinear Effects in a Hypersonic Wave. JETP Letters, Vol.4, Iss. 4, p.90-92, ISSN: 0021-3640.

Ready, J. F. (1971). Effects of High Power Laser Radiation, Academic, New York, London. 
Ritus, A. (1982). Study of Mandel'shtam-Brillouin light scattering in crystals and glasses respecting to problems of quantum electronics and fiber optics. Trudy FIAN 137, pp. 3-80.

Robinson M.M., Yan Y.-X., Gamble E.B., Williams Jr., L.R., Meth J.S, \& Nelson K.A. (1984). Picosecond impulsive stimulated Brillouin scattering: Optical excitation of coherent transverse acoustic waves and application to time-domain investigations of structural phase transitions. Chem.Phys. Lett., Vol.112, pp.491-496, ISSN 0009-2614.

Sakakura, M., Terazima, M., Shimotsuma, Y., Miura, K. \& Hirao, K, (2007) Observation of pressure wave generated by focusing a femtosecond laser pulse inside a glass Opt. Exp. 15, 5674-5686, ISSN 1094-4087.

Sharma B.S. \& Rieckhoff, K.E. (1970). Laser-induced dielectric breakdown and mechanical damage in silicate glasses. Can. J. Phys. Vol.48(10), 1178-1191, ISSN 0008-4204.

Starunov V.S. \& Fabelinskii I.L. (1970) Stimulated Mandel'shtam-Brillouin scattering and stimulated entropy (temperature) scattering of light. Sov. Phys. Usp., Vol.13, pp.428428. ISSN: 0038-5670.

Strekalov, V. (2000).Mechanical damage of transparent dielectrics by focused laser radiation J. Tech. Phys. Lett. Vol.26, No. 24, pp.19-22. ISSN: 0320 - 0116.

Stuart, B, Feit, M, Rubenchik, A., Shore, B. \& Perry, M. (1995). Laser-Induced Damage in Dielectrics with Nanosecond to Subpicosecond Pulses. Phys. Rev. Lett. Vol.74, pp.2248-2251, ISSN 0031-9007.

Suguira, H., Ikeda, R., Kondo, K. \& T. Yamadaya, T. (1997). Densified silica glass after shock compression. J. Appl. Phys., Vol.81, pp.1651-1656, ISSN 0021-8979.

Tychinskii, V. (2008). Super-resolution and singularities in phase images Phys.Usp. Vol.178, №11, pp. 1205-1214. ISSN: 0038-5670.

Zhu, T., Maris, H., \& Tauc, J. (1991). Attenuation of longitudinal-acoustic phonons in amorphous $\mathrm{SiO}_{2}$ at frequencies up to $440 \mathrm{GHz}$. Phys. Rev. B Vol.44, pp.4281-4289, ISSN 1098-0121.Dictionary - 


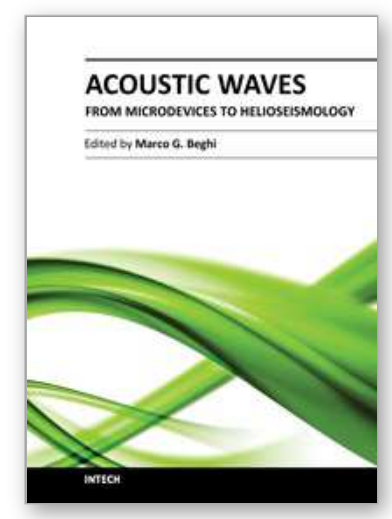

\author{
Acoustic Waves - From Microdevices to Helioseismology \\ Edited by Prof. Marco G. Beghi
}

ISBN 978-953-307-572-3

Hard cover, 652 pages

Publisher InTech

Published online 14, November, 2011

Published in print edition November, 2011

The concept of acoustic wave is a pervasive one, which emerges in any type of medium, from solids to plasmas, at length and time scales ranging from sub-micrometric layers in microdevices to seismic waves in the Sun's interior. This book presents several aspects of the active research ongoing in this field. Theoretical efforts are leading to a deeper understanding of phenomena, also in complicated environments like the solar surface boundary. Acoustic waves are a flexible probe to investigate the properties of very different systems, from thin inorganic layers to ripening cheese to biological systems. Acoustic waves are also a tool to manipulate matter, from the delicate evaporation of biomolecules to be analysed, to the phase transitions induced by intense shock waves. And a whole class of widespread microdevices, including filters and sensors, is based on the behaviour of acoustic waves propagating in thin layers. The search for better performances is driving to new materials for these devices, and to more refined tools for their analysis.

\title{
How to reference
}

In order to correctly reference this scholarly work, feel free to copy and paste the following:

N.E. Bykovsky and Yu.V. Senatsky (2011). Excitation of Periodical Shock Waves in Solid-State Optical Media (Yb:YAG, Glass) at SBS of Focused Low-Coherent Pump Radiation: Structure Changes, Features of Lasing, Acoustic Waves - From Microdevices to Helioseismology, Prof. Marco G. Beghi (Ed.), ISBN: 978-953-307-5723, InTech, Available from: http://www.intechopen.com/books/acoustic-waves-from-microdevices-tohelioseismology/excitation-of-periodical-shock-waves-in-solid-state-optical-media-yb-yag-glass-at-sbs-offocused-low

\section{INTECH}

open science | open minds

\section{InTech Europe}

University Campus STeP Ri

Slavka Krautzeka 83/A

51000 Rijeka, Croatia

Phone: +385 (51) 770447

Fax: +385 (51) 686166

www.intechopen.com

\section{InTech China}

Unit 405, Office Block, Hotel Equatorial Shanghai

No.65, Yan An Road (West), Shanghai, 200040, China 中国上海市延安西路65号上海国际贵都大饭店办公楼 405 单元

Phone: +86-21-62489820

Fax: $+86-21-62489821$ 
(C) 2011 The Author(s). Licensee IntechOpen. This is an open access article distributed under the terms of the Creative Commons Attribution 3.0 License, which permits unrestricted use, distribution, and reproduction in any medium, provided the original work is properly cited. 